\title{
METHODOLOGY FOR THE ANALYSIS OF POST-TENSIONED STRUCTURES USING A CONSTITUTIVE SERIAL-PARALLEL RULE OF MIXTURES
}

\author{
A. Cornejo ${ }^{1}$, L.G.Barbu ${ }^{2}$,C.Escudero ${ }^{2}$, X. Martínez ${ }^{2}$, S. Oller ${ }^{2}$ and A. H. Barbat ${ }^{2}$ \\ ${ }^{1}$ Centre Internacional de Mètodes Numèrics en Enginyeria (CIMNE), Campus Norte UPC, 08034 Barcelona, Spain \\ ${ }^{2}$ Universidad Politécnica de Cataluña (UPC), Campus Norte UPC, 08034 Barcelona, Spain
}

\begin{abstract}
The main purpose of this paper is to develop a reliable method based on a three-dimensional (3D) finiteelement (FE) model to simulate the constitutive behaviour of reinforced concrete structures strengthened with post-tensioned tendons taking into account the reduction of the pre-stressing stress due to the steel relaxation. The pre-tensioned concrete is modelled as a composite material whose behaviour is described with the serial-parallel rule of mixtures (S/P RoM) [1-3] whereas the stress relaxation of the steel is simulated using a viscoelastic model called Generalized Maxwell. A 3D FE model was used, where the nonlinear material behaviour and geometrical analysis based on incremental-iterative load methods were adopted. Validation by comparison with the analytic solution will be done for the case of a concrete beam with a parabolic pre-tensioned steel tendon embedded and some viscoelastic cases are performed in order to perceive the behaviour of the Generalized Maxwell model. Several examples are shown where the capabilities of the method on large scale structures are exhibited.
\end{abstract}

Keywords: Finite element method, post-tensioned, serial-parallel rule of mixtures, composites, viscoelasticity.

\section{Introduction and state of the art}

Pre-tensioning has been used successfully to improve the performance of existing reinforced concrete structures and high-strength tendons can be used to effectively increase their ultimate capacity.

One of the most commonly used methods to simulate the pre-stressed system (for straight and parabolic shaped tendons) consists in adding concentrated loads at the anchoring zones and an ascending distributed load that represents the effect of the curvature of the tendon along its path [4]. This method has the advantage that it is really easy to apply but is limited to simple geometries in which the equivalent system of forces can be estimated properly. Another more sophisticated method consists in a combination of the finite element method (that is used to model the concrete) and bi-articulated elements connecting pair of nodes of the FE mesh in order to represent the steel tendon [5][6][7].

Many attempts have been made to correctly model the behaviour of pretensioned concrete members using this second technique.

Arab et al. [8] compared several modelling techniques concerning the prestress tendons. One model uses an extrusion technique; a second model is built following the concept of embedded reinforcement in which the prestress tendons are implemented using one-dimensional elements which are embedded in the concrete continuum elements. For both models, bondslip behaviour is achieved by implementing the frictional nature at the concrete-strand interface. However, in the extruded model, this is achieved through contact surface algorithms while the embedded model uses nodal constraints and master-slave connections It is concluded that a correct assessment of the overall behaviour of the pre-tensioned elements can be achieved with both techniques

As stated by [7], a nonlinear finite element model using a commercial code (ANSYS) was used by Kaewunruen and Remennikov [9] to analyse railway prestressed concrete sleepers. This model included

\footnotetext{
${ }^{1}$ Corresponding author

e-mail address: acornejo@cimne.upc.edu (A. Cornejo)
} 
brick elements to represent the concrete matrix with embedded three-dimensional truss elements simulating the prestressing.

Stephen [10] used a comprehensive three-dimensional finite element model utilizing the commercial code (ABAQUS) to simulate the long-term behaviour of precast/prestressed concrete bridges. This model included elastoplastic material modelling capable of capturing the nonlinear behaviour of various concrete members (e.g., deck slab, pretensioned concrete girders) due to long term effects such as creep and shrinkage. The model required an external subroutine to facilitate specific operations such as prestressing and application of the long term effects.

Rabczuk and Eibl [11] proposed a coupled element free Galerkin method to analyse prestrsessed concrete beams under quasi-static loading. The constitutive law governing the concrete medium was based on a coupled damage-plasticity model. The reinforcement was modelled as discrete beam elements so that the interaction between concrete and reinforcement can be modelled. The bond model included two modes of failure: pullout failure, and splitting failure. The formulation of the bond model was based on the radial stress-radial strain relation with three distinct domains: the nonlinear material behaviour including the initiation and propagation of cracks, linear softening, and residual strength.

More recently, Ayoub and Filippou proposed a nonlinear model for simulation of the pretensioned prestressed concrete girders [12]. The modelling approach consisted of three main components: concrete girder simulated as a beam-column, prestressing strands modelled as truss elements, and a bond element to model the prestress transfer between the concrete and strands. The constitutive laws governing the nonlinear response of concrete and strands were based on discretization of the media into fibers with uniaxial hysteric models. The bond model at the interface between the concrete and strand was formulated using special bond stress-slip relations. The pretensioning mechanism was divided into discrete time steps representing various stages of the operation.

Another field where prestressing techniques are used is in the study of nuclear containments. For instance, Hu and Lin [13] conducted an analytical study also using ABAQUS finite element program to predict the ultimate pressure capacity of the PWR (Pressure Water Reactor) prestressed concrete containment at Maanshan nuclear power plant. The prestressing tendons and reinforcing steel were modelled as uni-axial materials that are smeared through the element section.

The previous method can represent the behaviour of the composite material but, implicitly, the finite element mesh is strongly dependent on the trajectory of the steel tendons since they connect pair of nodes of the finite element. This means that in zones with high curvatures of the tendons one must place a high density of finite elements, with the consequent increase of the computational cost. In addition, the friction or debonding effects between the concrete and steel cannot be easily included. These are the two main approaches found in literature.

The proposed formulation only requires a finite element mesh of any type and any spatial discretization and a linear description of the steel tendons. The pre-tensioned concrete is modelled as a composite material whose behaviour is described with the serial-parallel rule of mixtures ( $\mathrm{S} / \mathrm{P} \mathrm{RoM})$. The effective pre-tensioning stress is applied as an initial strain imposition only in the steel material used to model the tendons. By means of the S-P RoM [1,2] equilibrium is reached at each integration point between the passive and active steel and concrete and the strain tensor of the steel is updated with the contribution of the concrete. An important advantage of applying the proposed methodology is that one can assume that each material behaves following its own constitutive law (elasticity, damage, plasticity, viscoelasticity, etc.). Next, the displacement field is updated until the global convergence of forces is achieved. The methodology is valid for both straight and curvilinear steel tendons. Examples of both cases will be shown.

Regarding the stress relaxation of the active steel, the general methodology consists in applying analytical formulas found in the current design regulations [14] whose variables are the elastic properties of the steel and the initial stress applied and, of course, the time increments. These methods are applied by means of analytical expressions of the stress reduction that can be useful in simple calculations but are difficult to extend to the finite element method, which is the main feature of the proposed Generalized Maxwell viscoelastic model [15]. 
The suggested model is capable of predicting the stress tensor time evolution (triaxial stress state) with only two parameters: the viscoelastic coefficient and the delay time. Both parameters and the model are exhaustively described in this paper.

\section{Constitutive modelling of pre-stressed reinforced concrete with the Serial-Parallel rule of mix- tures}

The Serial-Parallel rule of mixtures (S-P RoM) defines two different compatibility conditions between the strain a stress states of the composite constituent materials: it states an iso-strain condition on the parallel direction, usually the fibre direction, and it defines an iso-stress condition on the serial direction, usually the remaining directions. Using these compatibility equations in a composite made of matrix and fibre, if the matrix structural capacity is lost due to excessive shear stresses, the iso-stress condition also reduces the shear capacity of fibre, and consequently the composite serial strength is also reduced. Since the behaviour of the composite is different depending on the serial or parallel direction, one must split the strain and stress tensors in its serial and parallel parts. This is done with two complementary fourth order projector tensors, one corresponding to the serial direction $\left(P_{S}\right)$ and the other to the parallel direction $\left(P_{P}\right)$. These tensors are defined from the fibre axial direction in the composite. Thus,

$$
\varepsilon=\varepsilon_{P}+\varepsilon_{S} \text { with } \varepsilon_{P}=P_{P}: \varepsilon \text { and } \varepsilon_{S}=P_{S}: \varepsilon
$$

Where

$$
P_{S}=I-P_{P} ; P_{P}=N_{P} \otimes N_{P} \text { and } N_{P}=e_{1} \otimes e_{1}
$$

Being $e_{1}$, the director vector that determines the parallel behaviour (fibre direction), and $I$ the identity. The stress state may be split analogously, finding its parallel and serial parts using also the 4th order tensors $P_{P}$ and $P_{S}$ :

$$
\sigma=\sigma_{P}+\sigma_{S} \text { with } \sigma_{P}=P_{P}: \sigma \text { and } \sigma_{S}=P_{S}: \sigma
$$

2.1. Main hypothesis for the numerical formulation

The S-P RoM assumes the following hypothesis in order to take into account the strain-stress states defined in the previous paragraphs:

1. The composite is composed by only two components: fibre and matrix

2. Component materials have the same strain in parallel (fibre) direction.

3. Component materials have the same stress in serial direction.

4. Composite material response is in direct relation with the volume fractions of compounding materials.

5. Homogeneous distribution of phases is considered in the composite.

6. Perfect bounding between components is considered.

\subsection{Constitutive models of simple materials (components)}

Each composite component material is computed with its own constitutive equation. So, the stresses in the matrix and fibre materials are obtained in the simplest case of linear elastic behaviour using:

$$
\begin{gathered}
{ }^{m} \sigma={ }^{m} C:{ }^{m} \varepsilon \\
{ }^{f} \sigma={ }^{f} C:{ }^{f} \varepsilon
\end{gathered}
$$

Where ${ }^{m} C$ and ${ }^{f} C$ are the elastic constitutive tensors in the matrix and fibre, respectively. The previous equations ca be rewritten taking into account the serial-parallel split of the strain and stress tensors: 


$$
\left[\begin{array}{l}
{ }^{i} \sigma_{P} \\
{ }^{i} \sigma_{S}
\end{array}\right]=\left[\begin{array}{ll}
{ }^{i} C_{P P} & { }^{i} C_{P S} \\
{ }^{i} C_{S P} & { }^{i} C_{S S}
\end{array}\right]:\left[\begin{array}{c}
{ }^{i} \varepsilon_{P} \\
{ }^{i} \varepsilon_{S}
\end{array}\right]
$$

Where

$$
\begin{array}{ll}
{ }^{i} C_{P P}=P_{P}:{ }^{i} C: P_{P} & { }^{i} C_{P S}=P_{P}::^{i} C: P_{S} \\
{ }^{i} C_{S P}=P_{S}::^{i} C: P_{P} & { }^{i} C_{S S}=P_{S}::^{i} C: P_{S}
\end{array}
$$

\subsection{Equilibrium and compatibility equations}

In order to state the stress equilibrium and the strain compatibility, one must analyse the hypothesis previously exposed:

$$
\begin{gathered}
\text { Parallel behaviour: } \begin{array}{c}
{ }^{c} \varepsilon_{P}={ }^{f} \varepsilon_{P}={ }^{m} \varepsilon_{P} \\
{ }^{c} \sigma_{P}={ }^{f} k \cdot{ }^{f} \sigma_{P}+{ }^{m} k \cdot{ }^{m} \sigma_{P}
\end{array} \\
{ }^{c} \varepsilon_{S}={ }^{f} k \cdot{ }^{f} \varepsilon_{S}+{ }^{m} k \cdot{ }^{m} \varepsilon_{S} \\
\text { Serial behaviour: }{ }^{c} \sigma_{S}={ }^{f} \sigma_{S}={ }^{m} \sigma_{S}
\end{gathered}
$$

where superscripts $c, m$ and $f$ stand for composite, matrix and fibre, respectively and ${ }^{i} k$ corresponds to the volume fraction coefficient of each constituent in the composite.

\subsection{Serial-Parallel Rule of Mixtures algorithm}

The known variable that enters the algorithm (input) is the strain state ${ }^{c} \boldsymbol{\varepsilon}$ of the composite material at time $t+\Delta t$. From this input, the S-P RoM has to find a pair of strain/stress tensors for each component that fulfils the equilibrium, compatibility and the constitutive equations in each integration point. The first thing done by the algorithm is to split the strain tensor into its parallel and its serial parts, in order to compute the strain state in the matrix and the fibre. The parallel strain component is, according to equation (7), the same for both materials and for the composite. On the other hand, the serial strain component requires a prediction of the strains expected in one of the composite components. If this prediction is done for the matrix (could be the fibre), the increment of its serial strains can be computed as:

$$
\left.\left[{ }^{m} \Delta \varepsilon_{S}\right]^{0}=A:\left[{ }^{f} C_{S S}:{ }^{c} \Delta \varepsilon_{S}+{ }^{f} k \cdot\left({ }^{f} C_{S P}-{ }^{m} C_{S P}\right)\right)^{c} \Delta \varepsilon_{P}\right]
$$

With $A=\left[{ }^{m} k \cdot{ }^{f} C_{S S}+{ }^{f} k \cdot{ }^{m} C_{S S}\right]^{-1}$ and ${ }^{m} \Delta \varepsilon_{S}={ }^{t+\Delta t}\left[{ }^{c} \varepsilon_{S}\right]-^{t}\left[{ }^{c} \varepsilon_{S}\right]$.

The initial approximation of the independent variable ${ }^{\boldsymbol{m}} \boldsymbol{\varepsilon}_{\boldsymbol{S}}$, proposed by Rastellini [1] and described in the equation (9), is computed considering that the distribution of total strain, in its parallel and serial parts. Its computation is done using the composite tangent stiffness matrix from the previous time step. With the prediction of the matrix serial strains, the fibre serial strains can be computed, in the iteration step $n$, according to equation (8), 


$$
{ }^{t+\Delta t}\left[{ }^{f} \Delta \varepsilon_{S}\right]^{n}={\frac{1}{f_{k}}}^{t+\Delta t}\left[{ }^{c} \varepsilon_{S}\right]-{\frac{}{{ }^{f} k} k}^{t+\Delta t}\left[{ }^{m} \varepsilon_{S}\right]^{n}
$$

Being ${ }^{t+\Delta t}\left[{ }^{m} \varepsilon_{S}\right]^{n}={ }^{t}\left[{ }^{m} \varepsilon_{S}\right]+\left[{ }^{m} \Delta \varepsilon_{S}\right]^{n}$.

Adding the serial and parallel components of the strain tensor (equation (3)) in each component, one can evaluate its own constitutive model and compute the integrated stress tensor for both materials and update their internal variables. Once one has obtained the integrated stress tensor for each component, the stress equilibrium must be evaluated:

$$
\left[\Delta \sigma_{S}\right]^{n}={ }^{t+\Delta t}\left[m \sigma_{S}\right]^{n}-{ }^{t+\Delta t}\left[{ }^{f} \sigma_{S}\right]^{n} \leq \text { toler }
$$

If the residual stress is lower than a certain tolerance, the equilibrium is achieved and the structural Newton-Raphson can continue. However, if equation (11) is not fulfilled, the initial approximation of the ${ }^{m} \varepsilon_{S}$ must be updated like in any Newton-Raphson strategy. Using this strategy, the update is made using the Jacobian of the residual forces. It is obtained deriving the residue function with respect to the unknown. According to Rastellini [1], the expression for the Jacobian is given as follows:

$$
J=\left[{ }^{m} C_{S S}^{t}\right]^{n}+\frac{{ }^{m} k}{{ }^{f} k} \cdot\left[{ }^{f} C_{S S}^{t}\right]^{n}
$$

and, the correction of the matrix serial strains becomes

$$
{ }^{t+\Delta t}\left[{ }^{m} \varepsilon_{S}\right]^{n+1}={ }^{t+\Delta t}\left[{ }^{m} \varepsilon_{S}\right]^{n}-J^{-1}:\left[\Delta \sigma_{S}\right]^{n}
$$

In order to obtain quadratic convergence of the S-P RoM algorithm, the Jacobian matrix must be obtained using the tangent constitutive tensors for the fibre and the matrix. Depending on the constitutive equation defined for each material, the constitutive tensor cannot be obtained analytically. Thus, in order to obtain a reliable algorithm, the expression of the tangent tensor is obtained numerically with the procedure shown in Martinez et al. [1][3].

\subsection{Singularities of the imposed strain procedure}

The previous numerical formulation is capable of simulating the behaviour of reinforced concrete as a composite material composed by concrete and passive steel. If one wants to take into account the case of the active steel, both for the pre and the post-tensioned case, it is necessary to rewrite the compatibility condition of the S-P RoM.

Essentially, the perfect adherence between the two materials cannot be achieved since the compatibility is not ensured.

6. Relative movement between the components is allowed if and only if an imposed strain condition exists over one of them.

Therefore, loss of adherence is allowed only in the presence of the imposed strain loading, a peculiar load due to the fact that it is applied only on a component of the composite material. This implies that its contribution is not quantified in the external forces vector and it is an auto balanced load.

Equations (7) must therefore be rewritten taking into account the imposition of an initial strain for the fiber in order to represent the pre-stressing or post-tensioning of the active steel. In the first iteration of the S-P RoM algorithm, the parallel component of the strain tensor of the active steel is fixed to the imposed pre-stress value. Knowing the composite stress tensor and the parallel component of the strain 
of the fibre, one can obtain the total strain tensor of the fibre and, depending on the desired fibre constitutive model, the integrated stress tensor is computed.

The resolution algorithm of the S-P RoM equilibrates the serial components at each integration point and with the integrated stresses the internal forces vector is assembled. At this point in the problem resolution, the parallel component of the fibre stresses has yet to be balanced. Its effect is quantified in the system of equations in the residual forces computed at the end of the first global iteration of the problem.

$$
\begin{aligned}
& { }^{m} \varepsilon_{P}={ }^{c} \varepsilon_{P} \\
& \text { incr }=1 ; \text { iter }=1 ; \quad{ }^{f} \varepsilon_{P}=\varepsilon_{\text {imp }} \\
& { }^{m} \varepsilon_{P}={ }^{c} \varepsilon_{P} \\
& \text { incr } \geq 1 ; \text { iter }>1 ; \quad \quad{ }^{f} \varepsilon_{P}={ }^{c} \varepsilon_{P}+\varepsilon_{\text {imp }}
\end{aligned}
$$

The residual forces computed from the unbalanced internal forces vector are used to update the nodal displacements and the composite strains at each integration point. In the second global iteration of the structure, the parallel component of the strains at layer level (that is the input of the S-P RoM) is equal to the matrix strain needed to accommodate the imposed fibre strain.

Therefore, in the first global iteration the active steel has its parallel strain component fixed at the level of strain associated to the desired pre-stressing force, while in the second iteration the fibre strain is balanced by the resulting compression in the concrete.

\section{Time-dependent models}

One of the behaviours responsible for the nonlinearity in the materials' response over the time field is due to viscoelasticity. Viscoelasticity studies the rheological behaviour of materials, in other words, behaviours affected by the course of time. There is extensive literature available on this subject, especially in books that deal with the influence of time on these materials [15].

These models have non-invertible constitutive laws; however, each one represents the implicit inverse form of the other, in other words, a delayed elasticity model is the inverse form of a relaxation model representation.

\subsection{Uniaxial representation}

In order to introduce the viscous model, one can start with its uniaxial version with the objective of simplifying the expressions and understanding the method. The multiaxial expressions are shown following this section. This formulation is useful and suitable for the representation of different types of viscous behaviours in solids.

The spring-damper scheme for the Generalized Maxwell model can be seen in the Fig. 1. 


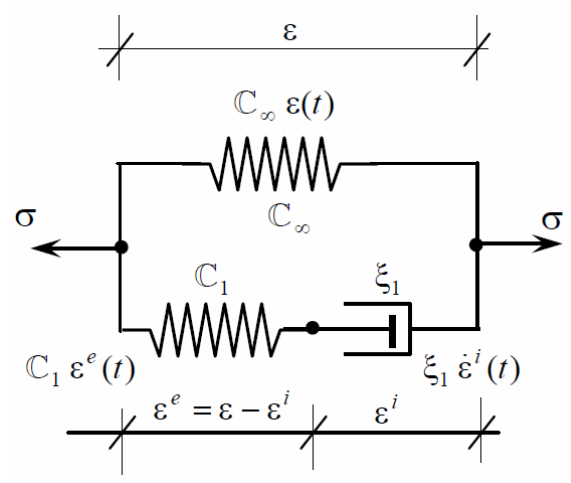

Fig. 1. The generalized Maxwell Model.

The stress state at any time is expressed as

$$
\left\{\begin{array}{l}
\sigma^{\infty}(t)=C_{\infty} \varepsilon(t) \\
\sigma^{i}(t)=C_{1}\left(\varepsilon(t)-\varepsilon^{i}(t)\right)=\xi_{1} \dot{\varepsilon}^{i}(t)
\end{array}\right.
$$

The equilibrium condition must meet the following relation,

$$
\sigma(t)=\sigma^{i}(t)+\sigma^{\infty}(t)=C_{1}\left(\varepsilon(t)-\varepsilon^{i}(t)\right)+C_{\infty} \varepsilon(t)=\xi_{1} \dot{\varepsilon}^{i}(t)+C_{\infty} \varepsilon(t)
$$

Stating that $\mathrm{C}_{0}=\mathrm{C}_{\infty}+\mathrm{C}_{1}$ and operating algebraically in the last equation, the stress equation is obtained as,

$$
\sigma(t)=\mathrm{C}_{0} \varepsilon(t)-\mathrm{C}_{1} \varepsilon^{i}(t)
$$

Using the equation (15), the following differential equation is obtained in the inelastic strain,

$$
\mathrm{C}_{1} \varepsilon(t)=\mathrm{C}_{1} \varepsilon^{i}(t)+\xi_{1} \dot{\varepsilon}^{i}(t) \Rightarrow \frac{\varepsilon(t)}{r_{1}}=\frac{\varepsilon^{i}(t)}{r_{1}}+\dot{\varepsilon}^{i}(t)
$$

Applying a strain $\varepsilon(t)$, from $t>\tau_{0}$, the differential equation can be solved for $\varepsilon^{i}(t)$ as,

$$
\begin{cases}\varepsilon^{i}(t)=0 & \forall \tau<\tau_{0} \\ \varepsilon^{i}(t)=\int_{-\infty}^{t} \frac{1}{r_{1}} \mathrm{e}^{-(t-s) / r_{1}} \cdot \varepsilon(s) d s & \forall \tau \geq \tau_{0}\end{cases}
$$

Substituting this equation into the (17), the following expression is obtained,

$$
\begin{cases}\sigma(t)=0 & \forall \tau<\tau_{0} \\ \sigma(t)=\mathrm{C}_{0} \varepsilon(t)-\frac{\mathrm{C}_{1}}{r_{1}} \int_{-\infty}^{t} \mathrm{e}^{-(t-s) / r_{1}} \cdot \varepsilon(s) d s & \forall \tau \geq \tau_{0}\end{cases}
$$


Defining now the uniaxial relaxation function $G(t)$, as the inverse of the uniaxial creep function,

$$
G(t)=[J(t)]^{-1}=\mathrm{C}_{\infty}+\mathrm{C}_{1} \mathrm{e}^{-t / r_{1}}
$$

Assuming the relaxation function inversion for this particular model, the following uniaxial creep function is obtained,

$$
J(t)=\frac{1}{\mathrm{C}_{\infty}}\left[1-\frac{\mathrm{C}_{1}}{\mathrm{C}_{0}} \mathrm{e}^{-\left(\frac{\mathrm{C}_{\infty}}{\mathrm{r}_{1} \mathrm{C}_{0}}\right) t}\right]
$$

And integrating the equation (20) by parts, the stress can be rewritten as the following compact form,

$$
\begin{cases}\sigma(t)=0 & \forall \tau<\tau_{0} \\ \sigma(t)=\int_{-\infty}^{t} G(t-s) \cdot \frac{d \varepsilon(s)}{d s} d s & \forall \quad \tau \geq \tau_{0}\end{cases}
$$

The stress expressed as shown above is the strain inverse function,

$$
\sigma(t)=\int_{-\infty}^{t} G(t-s) \cdot \frac{d \varepsilon(s)}{d s} d s \Rightarrow \varepsilon(t)=[\sigma(t)]^{-1}=\int_{-\infty}^{t} J(t-s) \cdot \frac{d \sigma(s)}{d s} d s
$$

In the particular case that the imposed strain $\varepsilon(t)$ meets the following simplification (see Fig. 2)

$$
\begin{cases}\varepsilon(t)=0 & \forall t<\tau_{0}=0 \\ \varepsilon(t)=\varepsilon_{0} & \forall t \geq \tau_{0}=0\end{cases}
$$

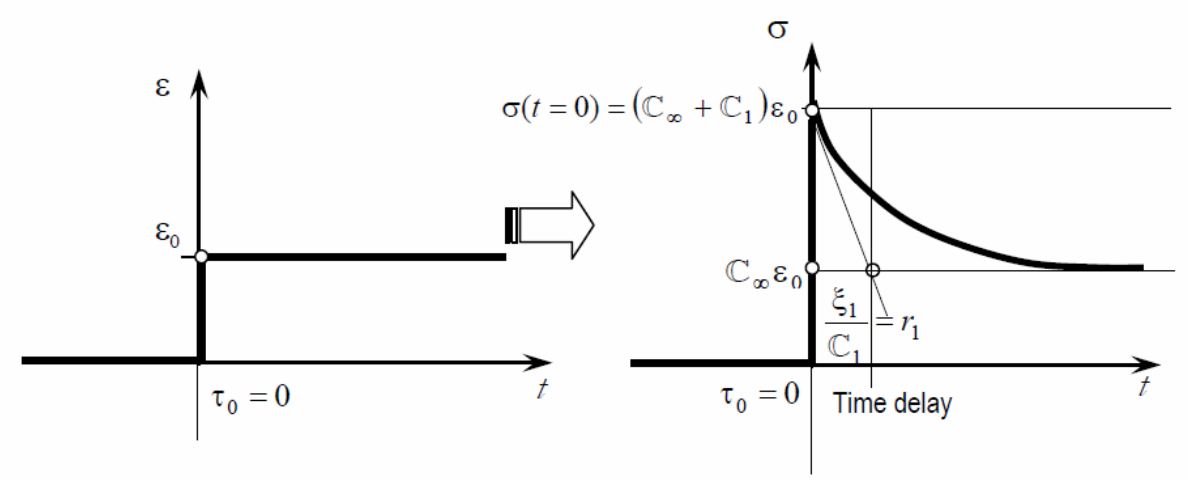

Fig. 2. Response of the generalized Maxwell Model under constant deformation.

The stress expression is reduced to the following simple form, 


$$
\begin{cases}\sigma(t)=0 & \forall \tau<\tau_{0} \\ \sigma(t)=\left(\mathrm{C}_{\infty}+\mathrm{C}_{1} \mathrm{e}^{-t / r_{1}}\right) \varepsilon_{0} & \forall \tau \geq \tau_{0}\end{cases}
$$

3.2. Multiaxial generalization of the Generalized Maxwell viscoelastic law

In this model, the multiaxial expression for stress, equation (20) is written at $\boldsymbol{t}+\boldsymbol{\Delta t}$ through the following approximation,

$$
\sigma_{i j}(t+\Delta t)=\mathrm{C}_{i j k l}\left[\varepsilon_{i j}(t+\Delta t)-\frac{\mathrm{C}_{1}}{\mathrm{C}_{0} \xi} \int_{-\infty}^{t+\Delta t} \mathrm{e}^{-(t+\Delta t-s) / r_{1}} \cdot \varepsilon_{k l}(s) d s\right]
$$

in which the variables involved are the ones already defined for the uniaxial problem section. The integral solution can be carried out without a convolution, it is then rewritten as,

$$
\begin{gathered}
\sigma_{i j}(t+\Delta t)=\mathrm{C}_{i j k l} \varepsilon_{i j}(t+\Delta t)-\left[\mathrm{C}_{i j k l} \frac{\mathrm{C}_{1}}{\mathrm{C}_{0} \xi} \int_{-\infty}^{t} \mathrm{e}^{-(t-s) / r_{1}} \cdot \varepsilon_{k l}(s) d s\right]- \\
-\mathrm{C}_{i j k l} \frac{\mathrm{C}_{1} \mathrm{C}_{0} \xi}{\int_{t}^{t+\Delta t}} \mathrm{e}^{-(t+\Delta t-s) / r_{1}} \cdot \varepsilon_{k l}(s) d s
\end{gathered}
$$

After integrating the third term using the trapezoidal rule and reordering the expressions, the stress is written as,

$$
\begin{aligned}
\sigma_{i j}(t+\Delta t)= & \mathrm{C}_{i j k l} \varepsilon_{i j}(t+\Delta t)-\mathrm{C}_{i j k l} \cdot \varepsilon_{k l}(t) \cdot \mathrm{e}^{-(\Delta t) / r_{1}}-\sigma_{i j}(t) \cdot \mathrm{e}^{-(\Delta t) / r_{1}}- \\
& -\mathrm{C}_{i j k l} \frac{\mathrm{C}_{1}}{\mathrm{C}_{0} \xi}\left[\mathrm{e}^{-(\Delta t) / r_{1}} \cdot \varepsilon_{k l}(t)-\varepsilon_{k l}(t+\Delta t)\right] \cdot \frac{\Delta t}{2} \\
\sigma_{i j}(t+\Delta t)= & -\mathrm{C}_{i j k l} \varepsilon_{k l}(t) \cdot \mathrm{e}^{-(\Delta t) / r_{1}}\left[1+\frac{\mathrm{C}_{1}}{\mathrm{C}_{0} \xi} \frac{\Delta t}{2}\right]+\mathrm{C}_{i j k l} \varepsilon_{k l}(t+\Delta t) \cdot\left[1-\frac{\mathrm{C}_{1}}{\mathrm{C}_{0} \xi} \frac{\Delta t}{2}\right]+ \\
& +\sigma(t) \cdot \mathrm{e}^{-(\Delta t) / r_{1}}
\end{aligned}
$$


A possible algorithm to solve the problem is shown in Table 1.

1- Obtaining the strain

$$
\left[\varepsilon_{i j}\right]^{t+\Delta t}
$$

2- Stress integration

$$
\left[\sigma_{i j}\right]^{t+\Delta t}=\left[\sigma_{i j}\right]^{t} \cdot \mathrm{e}^{-(\Delta t) / r_{1}}-\mathrm{C}_{i j k l}\left[\varepsilon_{k l}\right]^{t} \cdot \mathrm{e}^{-(\Delta t) / r_{1}}\left[1+\frac{\mathrm{C}_{1}}{\mathrm{C}_{0} \xi} \frac{\Delta t}{2}\right]+\mathrm{C}_{i j k l}\left[\varepsilon_{k l}\right]^{t+\Delta t} \cdot\left[1-\frac{\mathrm{C}_{1}}{\mathrm{C}_{0} \xi} \frac{\Delta t}{2}\right]
$$

Table 1. Algorithm for obtaining the stress of the generalized Maxwell model

\section{Validation of the formulation}

In the next section, some application examples of the S-P RoM are presented. The two first examples neglect the viscoelasticity and are compared with their analytical solution due to their simplicity. The rest of the examples include the time dependency due to the use of the Generalized Maxwell model for the behaviour of the active steel in the simulations.

\subsection{Pre-stressed beam with a linear centred tendon}

In this case, a $7 \mathrm{~m}$ length beam with a $1 \mathrm{~m}$ quadrangular cross section has been analysed. Inside the beam there is an adherent linear steel tendon whose area, As, is $0.04 \mathrm{~m}^{2}$. This means that the steel volumetric participation is equal to $4 \%$. The geometry of the beam can be seen in the Fig. 3. In this example the effect of the self-weight has been neglected and only one of the tips of the beam has been clamped.

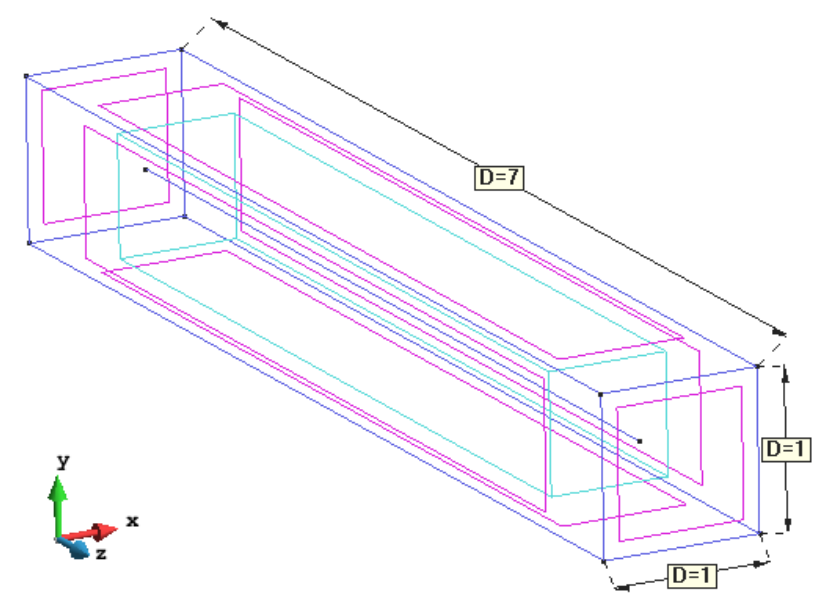

Fig. 3. Geometry of the beam and the steel tendon

The geometry of the Fig. 3 has been meshed with 7000 linear hexahedral finite elements (see Fig. 4). The steel tendon has been pre-stressed up to $1176 \mathrm{Mpa}$, which corresponds to an imposed strain, $\varepsilon_{\mathrm{s}}$, equal to 0.0056 . The young modulus of the steel, $\mathrm{E}_{\mathrm{s}}$, has been assumed equal to $210000 \mathrm{MPa}$ and the one of the concrete, $\mathrm{E}_{\mathrm{c}}, 35000 \mathrm{MPa}$.

The axial force in the steel tendon can be computed using (30). On the other hand, the elastic shortening of the concrete is obtained by means of (31). Finally, the theoretical longitudinal displacement can be 
computed using (32). The finite elements intersected by the steel tendon linear curve can be seen in the Fig. 5. Those elements are going to be pre-stressed since they have a certain volumetric fraction of active steel on which the imposed strain is applied.

$$
\begin{gathered}
N_{t}=A_{s} \sigma_{t}=4.704 \cdot 10^{7} \mathrm{~N} \\
\Delta \varepsilon=-\frac{N_{t}}{E_{c} A_{c}+E_{s} A_{s}}=-1.12 \cdot 10^{-3} \\
\delta_{\text {long }}=\Delta \varepsilon \cdot L=-7.840 \cdot 10^{-3} \mathrm{~m}
\end{gathered}
$$

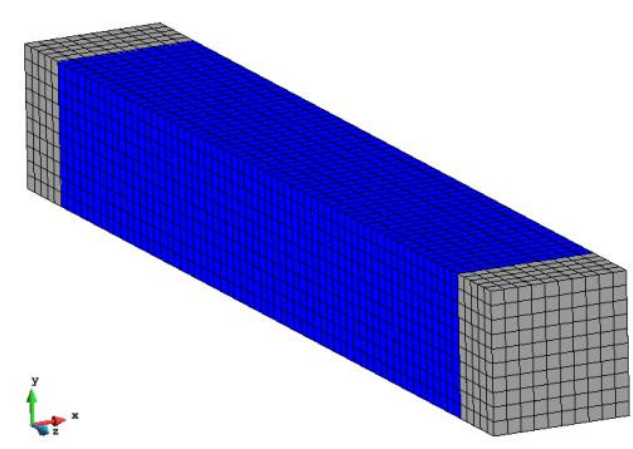

Fig. 4. Finite element mesh with 7000 linear hexahedra

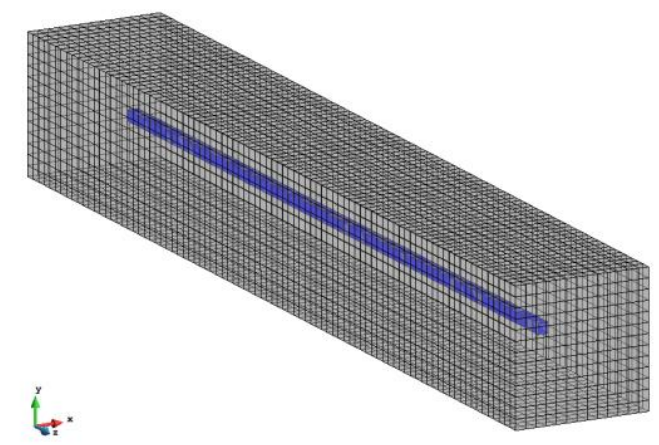

Fig. 5. Finite elements intersected by the steel tendon

Performing the calculation by means of the finite element code PLCd [16], in which this formulation has been implemented, one can obtain the displacement field shown in the Fig. 6. As can be seen, the simulated longitudinal displacement is equal to $7.83 \cdot 10^{-3} \mathrm{~m}$ while the analytical displacement was $7.84 \cdot 10^{-3} \mathrm{~m}$. This demonstrates the good agreement between the numerical formulation and the analytical solution. 


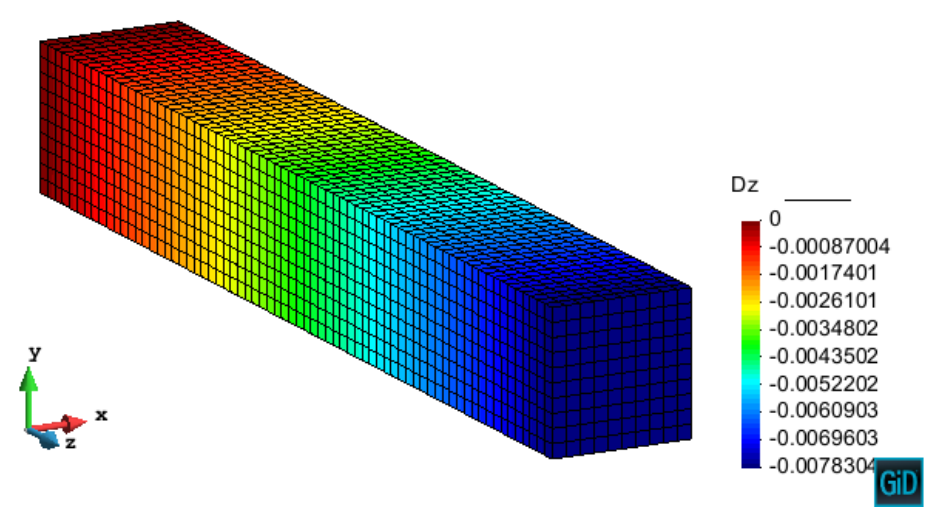

Fig. 6. Finite elements intersected by the steel tendon

\subsection{Pre-stressed beam with a parabolic steel tendon}

In this paragraph, the behaviour of a simply supported beam with an embedded parabolic steel tendon has been analysed. The beam has a length, $\mathrm{L}$, of $10 \mathrm{~m}$ and a height/width of $1 \mathrm{~m}$ as shown in the Fig. 7 . In Fig. 8 one can see the local axes of the active steel of each composite material. The most important issue to be noted in Fig. 8 is the coherence of the longitudinal axis of the composite component representing steel. In Fig. 9. are shown the different composite materials used in the calculation, each one symbolized with a different colour.

The steel tendon has been pre-stressed with a force, $\mathrm{P}$, of $5000 \mathrm{kN}$ and has an area equal to $0.005 \mathrm{~m}^{2}$. This implies that the stress reaches the $1000 \mathrm{Mpa}$. Regarding the concrete properties, the Young modulus, Ec, has been considered equal to $35875 \mathrm{Mpa}$.

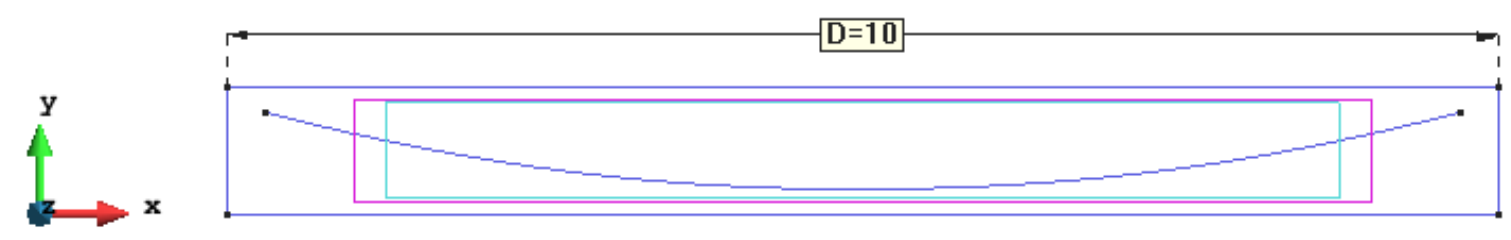

Fig. 7. Geometry of the beam and the parabolic steel tendon

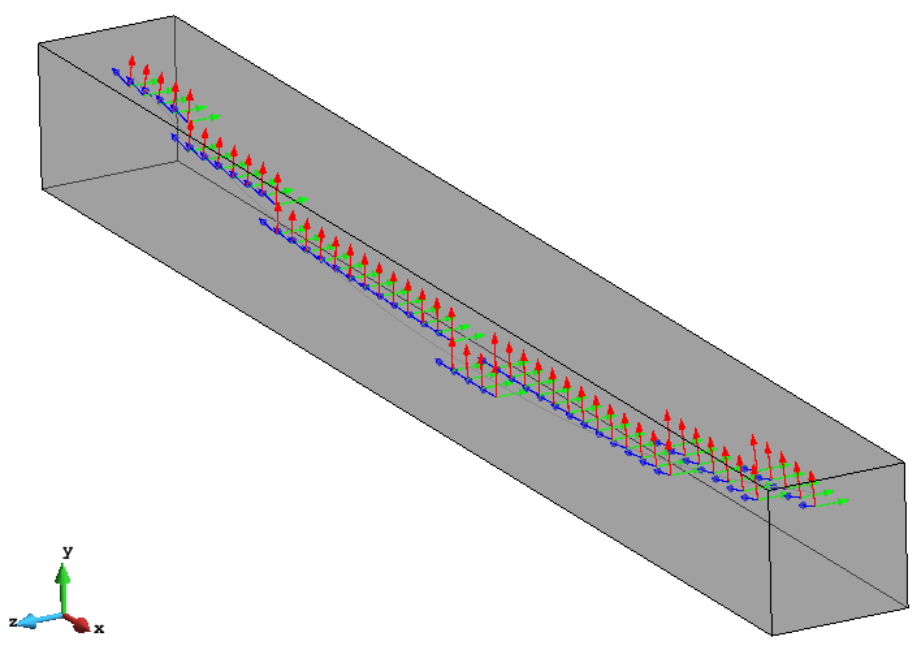

Fig. 8. Local axes of the composite materials intersected by the steel tendon 


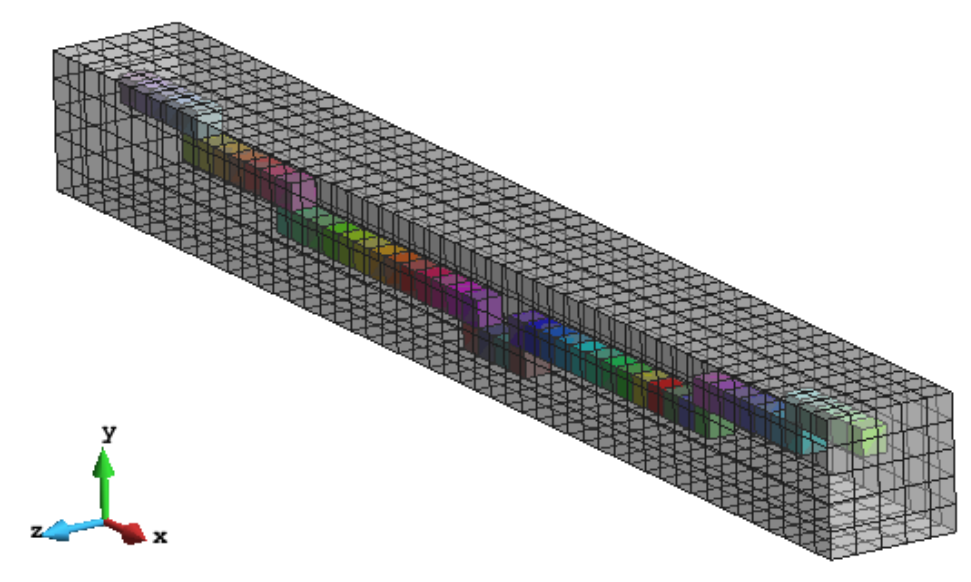

Fig. 9. Finite element mesh (1288 finite elements) and composite materials generated

In order to perform the analytic calculation, one must simplify the structural system as shown in the Figs. 10 and 11. As can be seen in these figures, the steel tendon induces two concentrated loads on the anchoring zones as well as two bending moments due to its eccentricity $(0.3 \mathrm{~m})$ with respect to the center of gravity of the section. In addition, due to the curvature of the steel tendon, an ascendant uniformly distributed load, $\eta$, must be included to take this effect into account. To obtain the value of $\eta$ one can use the equation (33).

$$
\eta=\frac{8 P\left(e_{1}+e_{2}\right)}{L^{2}}=240 \frac{k N}{m}
$$
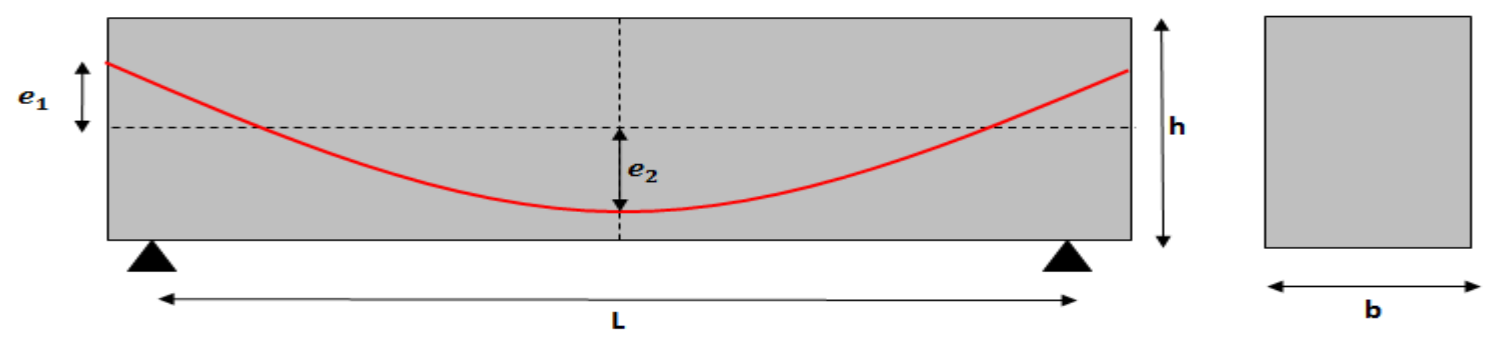

Fig. 10. Simplified structural scheme of the analyzed beam

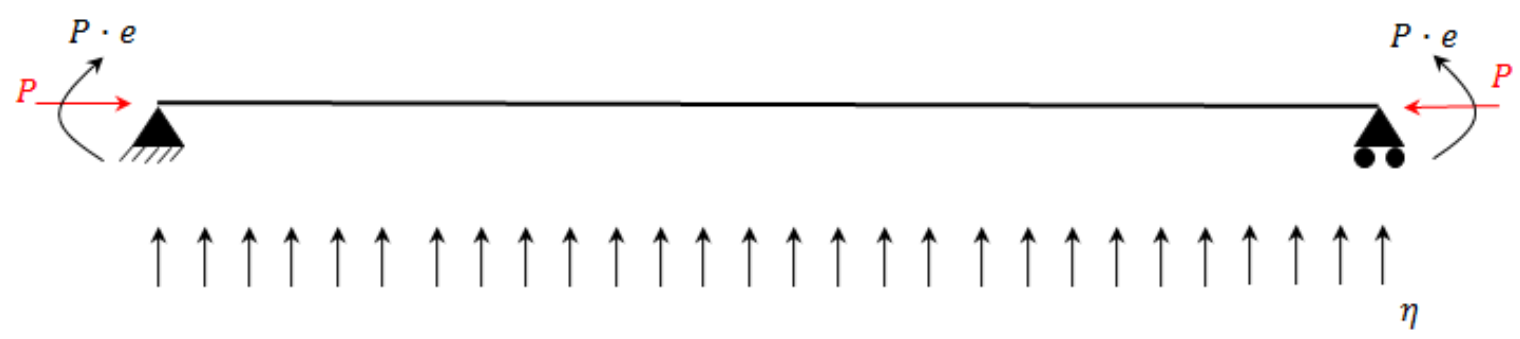

Fig. 11. Equivalent system of forces to simulate the pre-stressing system

In order to obtain the analytical vertical displacement, by means of analytical expressions and load superposition, the expression (34) can be derived. It is important to mention that, in general, the analytical formulae omit the effect of the shear deformation and, in addition, the elastic shortening of the concrete has been neglected. 


$$
\delta=\frac{5 \eta L^{4}}{384 E_{h} I}-\frac{3 P \cos (\phi) e_{1} L^{2}}{24 E_{h} I}=4.38 \mathrm{~mm}
$$

Where $E_{h}$ corresponds to the Young modulus of the homogenized section, $I$ the inertia of the section and, lastly, $\phi$ is the angle of the steel tendon on the anchoring zone.

Using the finite element code PLCd, one can obtain the displacement field shown in the Fig. 12 with a maximum displacement of $4.60 \mathrm{~mm}$.

Comparing the simulated results with the analytical one (equation 34), the difference between them is lower than the 5\% assuming the analytical solution as an exact solution (which is not entirely true). The longitudinal stress field in each material can be analysed in the Figs. 13 and 14. As can be seen in the previous figures, the pre-stressing system induces a bending state that generates tension stresses on the upper part of the beam and compression on the lower part, as expected. The stress field in the active steel can be seen in the Fig. 14. As expected, all the finite elements with active steel are fully tensioned with a value close to the imposed one (around $1000 \mathrm{Mpa}$ ). The variation of the tension stress in the active steel is due to the accommodation of the pre-stressing force by the concrete, the so-called elastic shortening.

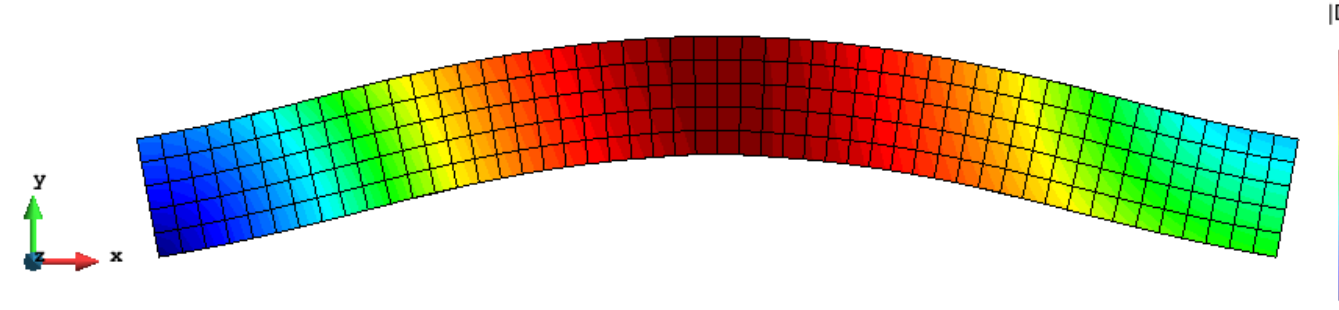

Fig. 12. Displacements field obtained with PLCd
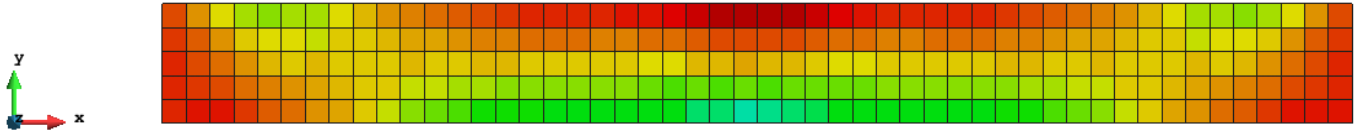

Sxx

Fig. 13. Stress field in the concrete obtained with PLCd
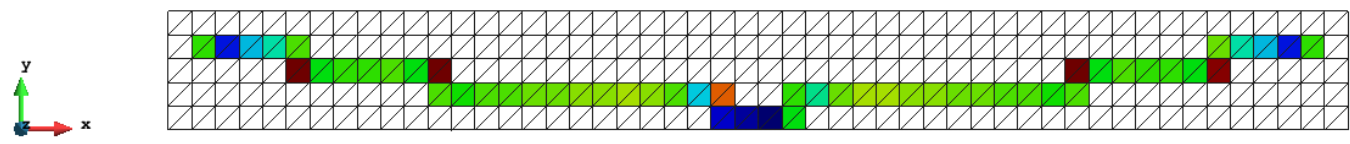

Fig. 14. Stress field in the active steel obtained with PLCd 


\subsection{Linear Beam taking into account Viscoelasticity}

In this case, a linear $1.5 \mathrm{~m}$ length beam with a typical industrial cross section has been analysed. There are two linear steel tendons embedded in the concrete pre-stressed up to $1092.84 \mathrm{MPa}$ which corresponds to a strain equal to 0.0056 . The Young modulus of the steel has been considered equal to $195150 \mathrm{MPa}$ and for the concrete is equal to $40200 \mathrm{MPa}$. The Poisson ratio is equal to 0.3 for the steel and 0.2 for the concrete. The viscous parameter has been considered equal to 0.15 and the delay time is 5 years. The geometry of the beam and its cross section can be seen in the Figs. 15 and 16.

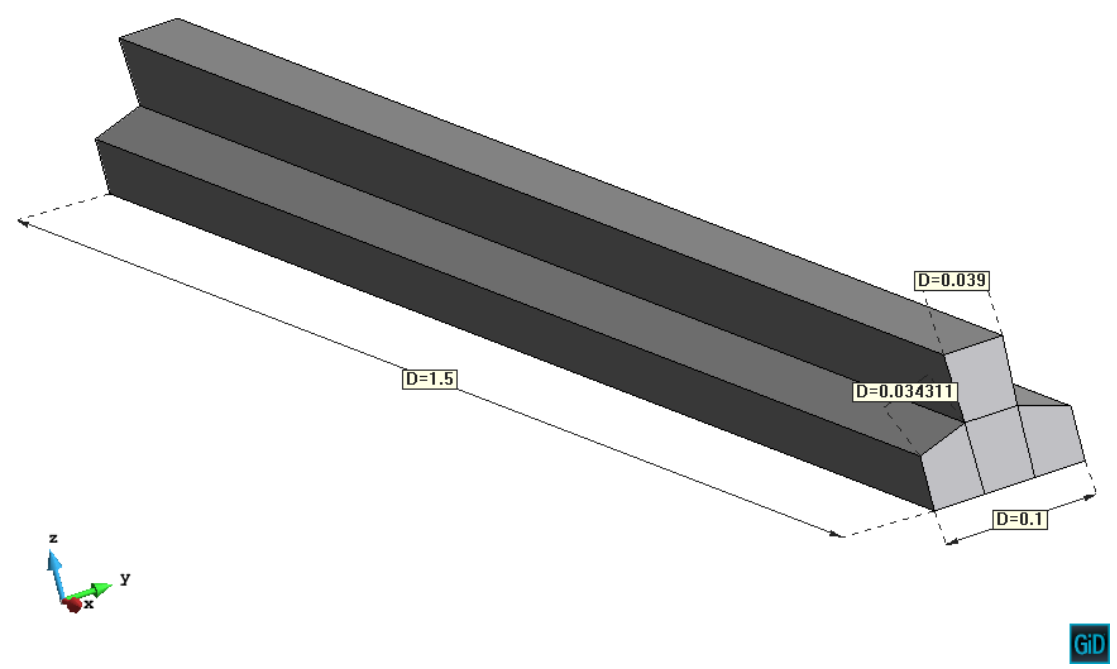

Fig. 15. Geometry of the beam

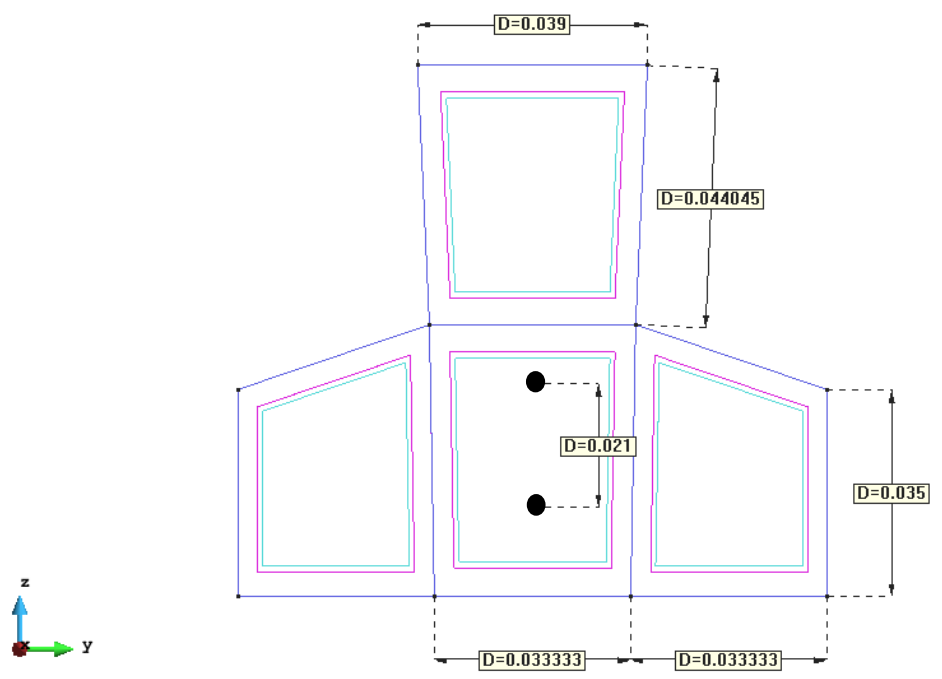

Fig. 16. Cross section of the beam

The structured finite element mesh can be seen in the Fig. 17. This mesh is composed by 12750 linear hexahedral finite elements and 16308 nodes. The finite elements intersected by the steel tendons can be seen in Fig.18. For this simple case, only two composite materials were generated since the entrance angle and volumetric participation along the finite elements intersected by each tendon is constant. This means that each finite element intersected by the steel tendon has a certain volumetric participation of active steel, on which the imposed strain procedure (see section 2.5) is going to be applied. 


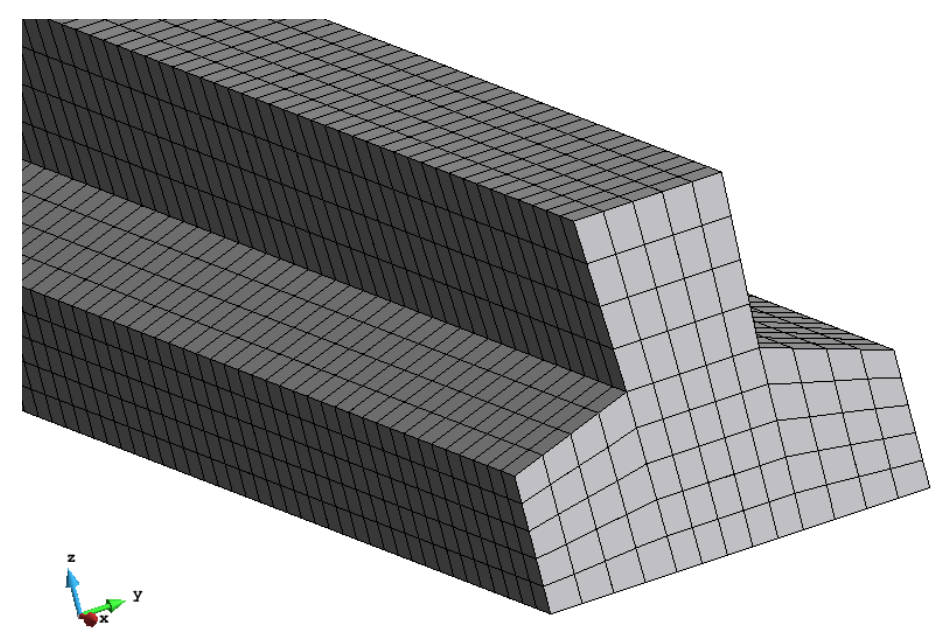

Fig. 17. Finite element mesh used (12750 finite elements)
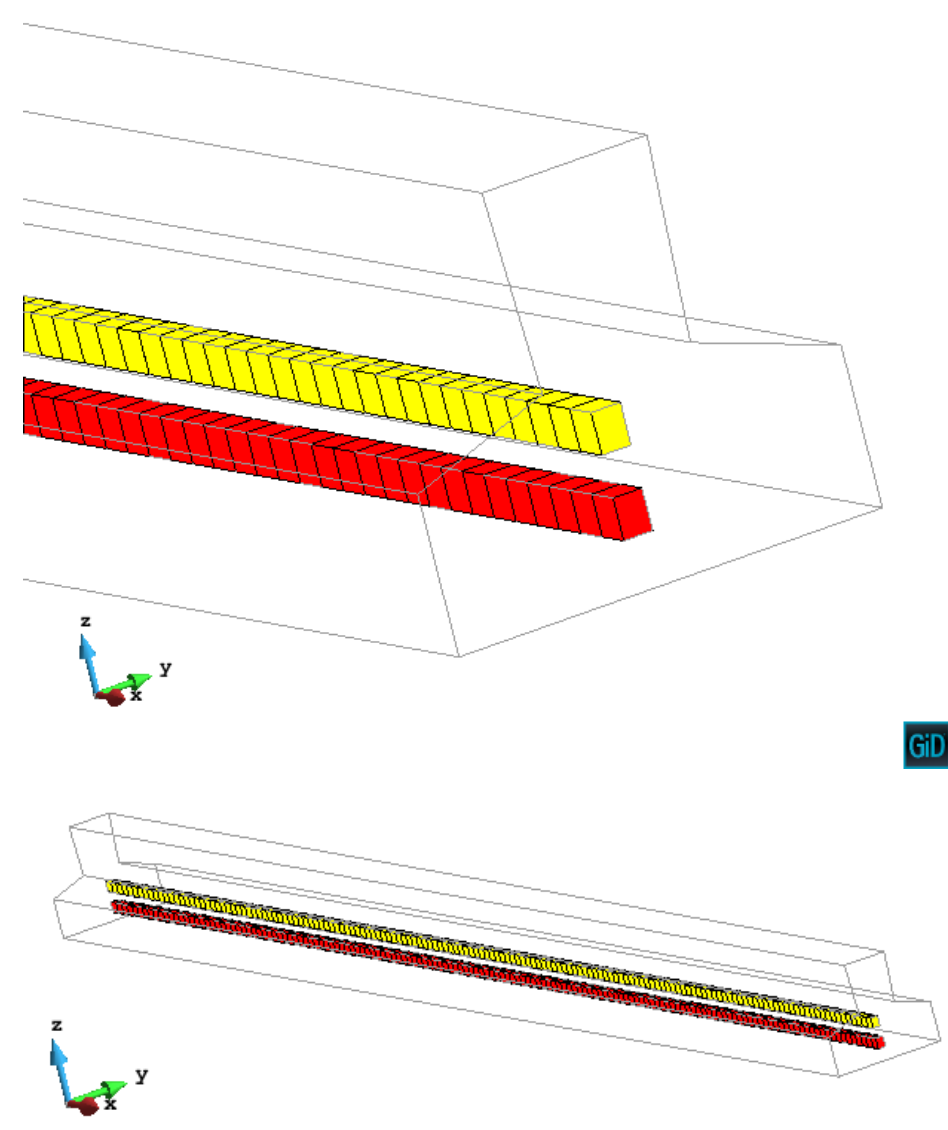

Fig. 18. Finite elements intersected by the steel tendon 
The strain was imposed at the level of 0.0056 for the active steel contained in those finite elements that were intersected by the tendons. Afterwards, five steps corresponding to $\Delta t_{\text {step }}=1$ year have been run. In Fig. 19. the displacement path for the first step can be seen while in the Fig. 20, 21 are shown the displacement paths for the $5^{\text {th }}$ year, when some stress relaxation has occurred.

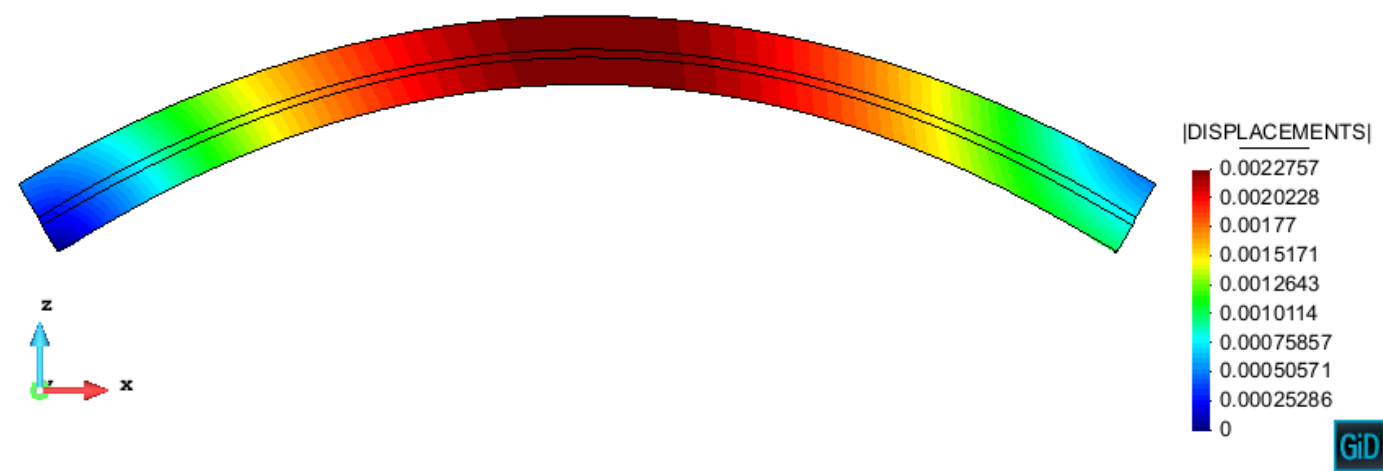

Fig. 19. Displacement path for $\mathrm{t}=0$ years, amplified 100 times.

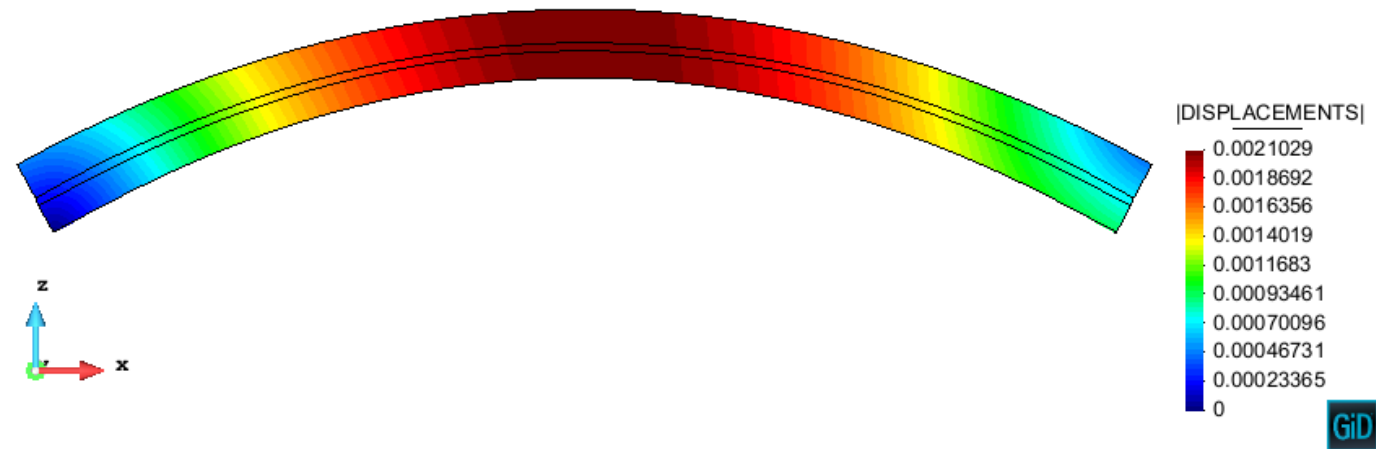

Fig. 20. Displacement path for $\mathrm{t}=5$ years, amplified 100 times.

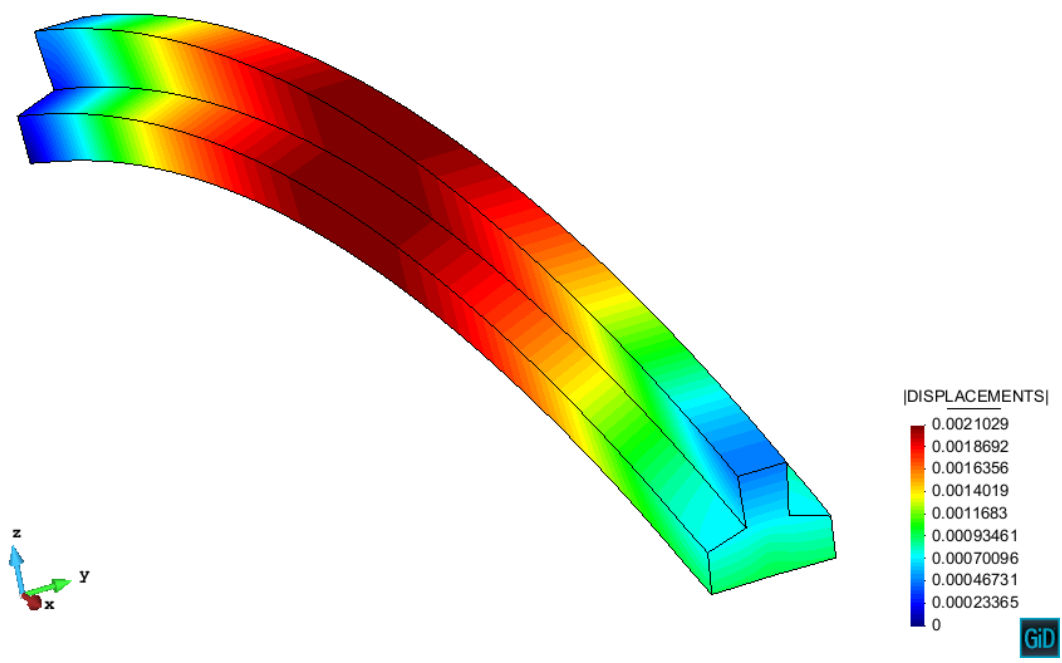

Fig. 21. Perspective of the displacement path for $\mathrm{t}=5$ years, amplified 50 times. 
In the Figs. 22-25 are shown the horizontal stresses for the concrete and steel for the first year and the year 5 . As can be seen in the previous pictures, the steel is fully tensioned with a peak value lower than the imposed one due to the elastic shortening of the concrete that has been taken into account implicitly in the finite element analysis. In addition, one can see how the minimum stress level is achieved in the tips of the beams.

For the concrete, as depicted in the Figs. 23 and 25, it is clear that the beam is compressed in the lower fiber whereas the upper fiber is tensioned, which compensates the effect of the self-weight in the case that we should take it into account. In the Fig. 26 is depicted the evolution of the displacement along time for a node located in the center of the beam. In this figure one can perceive the displacement reduction due to the stress relaxation of the active steel.

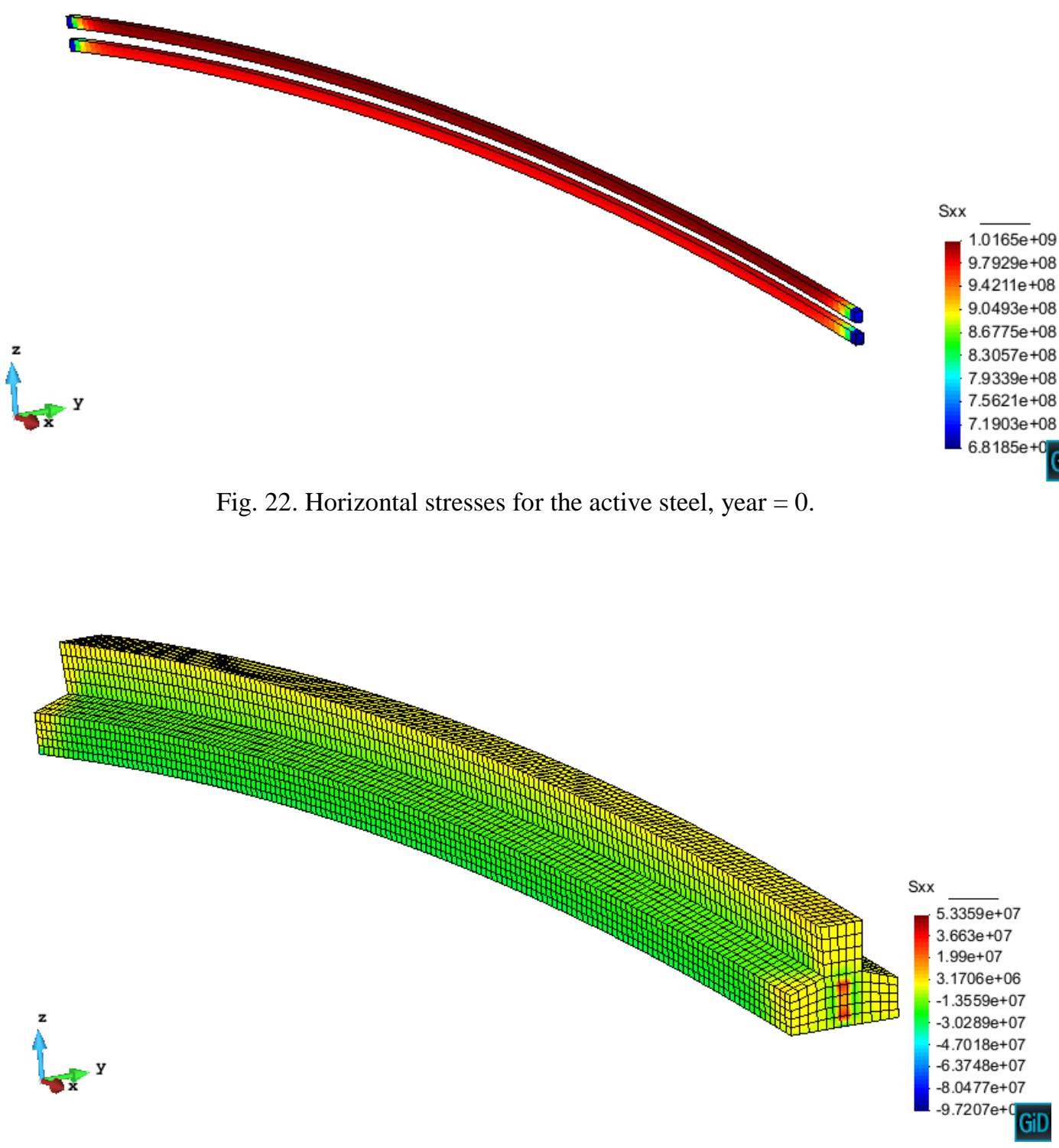

Fig. 23. Horizontal stresses for the concrete, year $=0$. 


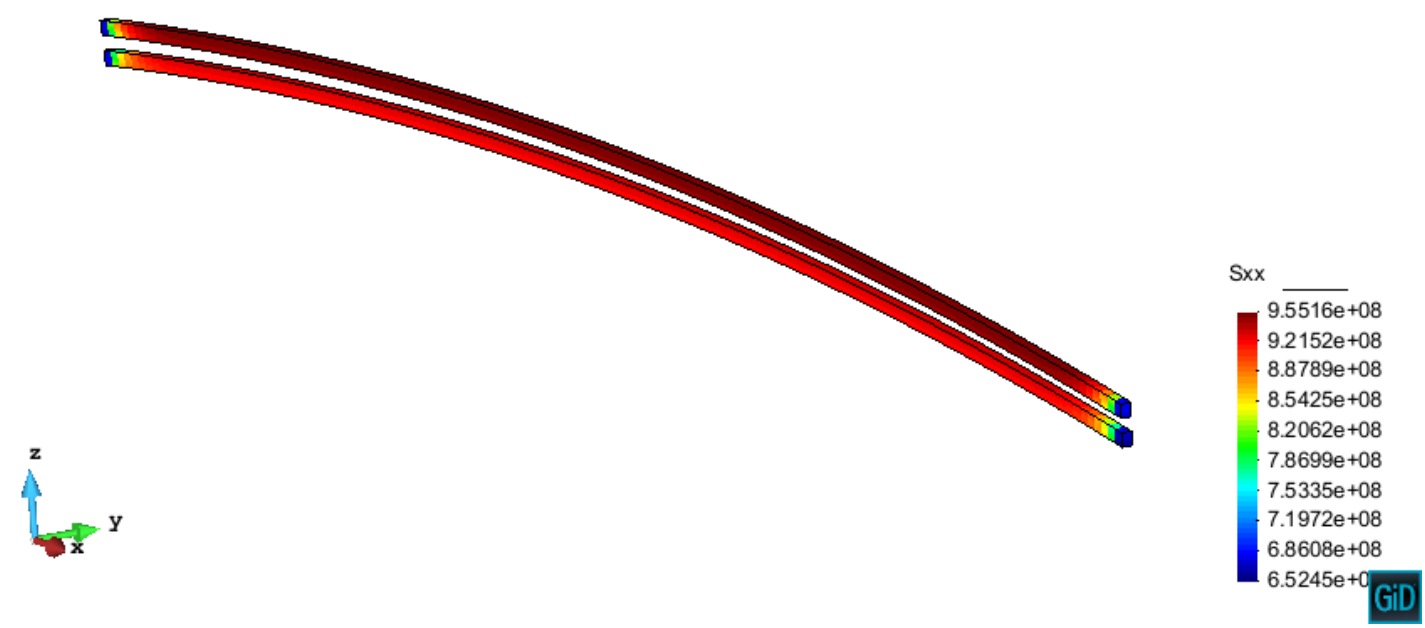

Fig. 24. Horizontal stresses for the active steel, year $=5$.

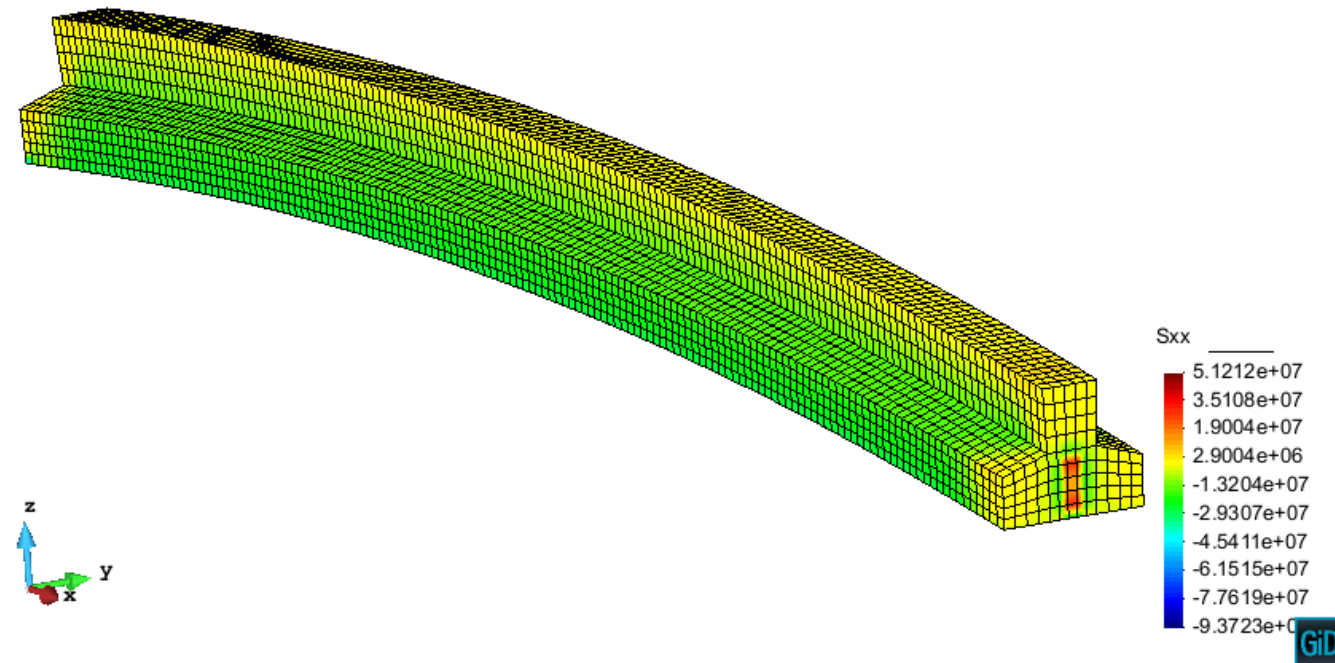

Fig. 25. Horizontal stresses for the concrete, year $=5$.

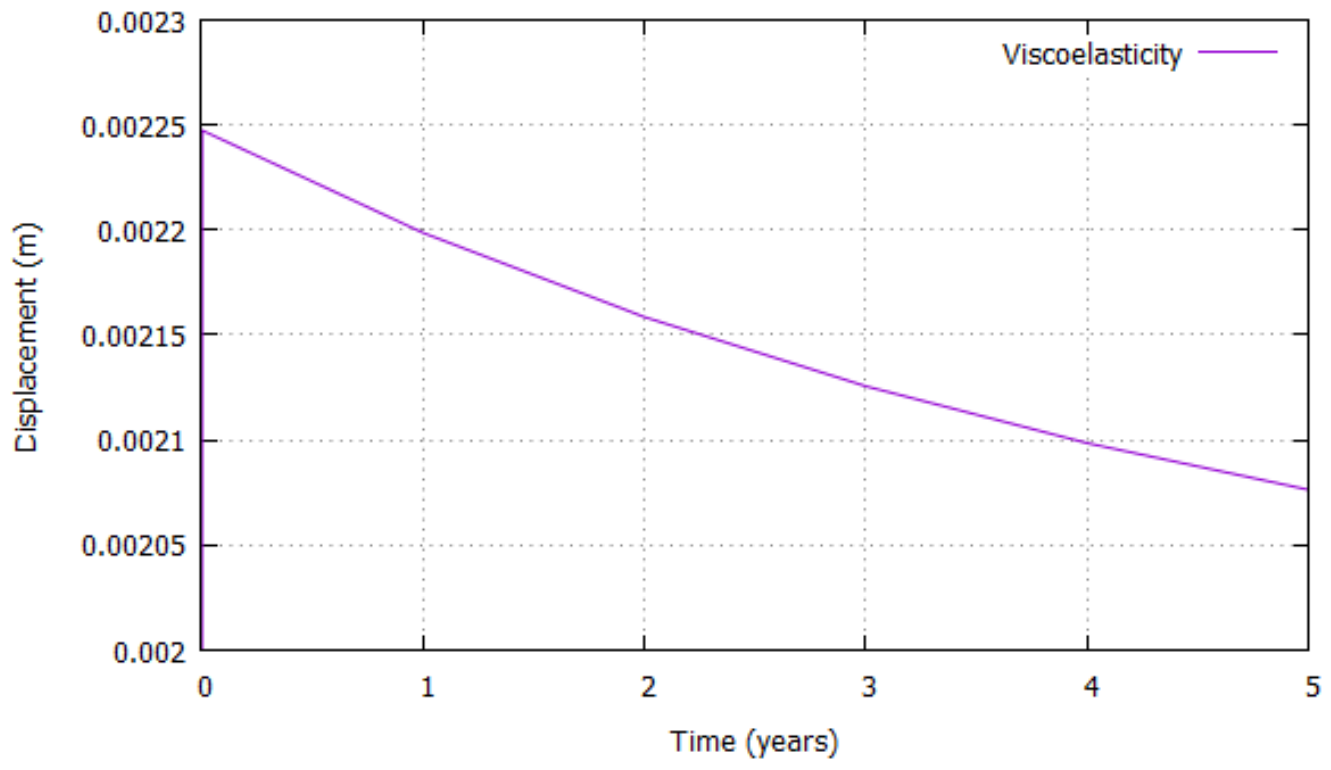

Fig. 26. Vertical displacement evolution along time for a centered node of the beam 


\subsection{Pre-stressed cantilever with six curvilinear steel tendons}

In this paragraph, a bridge in a progressive cantilever assembly has been analysed. This structure has a variable height and the central zone is made out of massive concrete to increase its stiffness. As can be seen in the Fig. 27, the central zone has a width of $1.5 \mathrm{~m}$ whereas the total span of the structure is 5.75 $\mathrm{m}$ with a minimum height of $1.9 \mathrm{~m}$. Through the walls of the cantilever, there are 6 steel tendons ( 3 in each vertical wall) with a curvilinear shape to increase the pre-stressing efficiency. All the steel tendons have a Young modulus equal to $195000 \mathrm{MPa}$ and have been pre-stressed up to $1092 \mathrm{MPa}$. The concrete has a Young modulus of $40200 \mathrm{MPa}$. The viscous parameter has been considered equal to 0.15 and the delay time is 5 years.

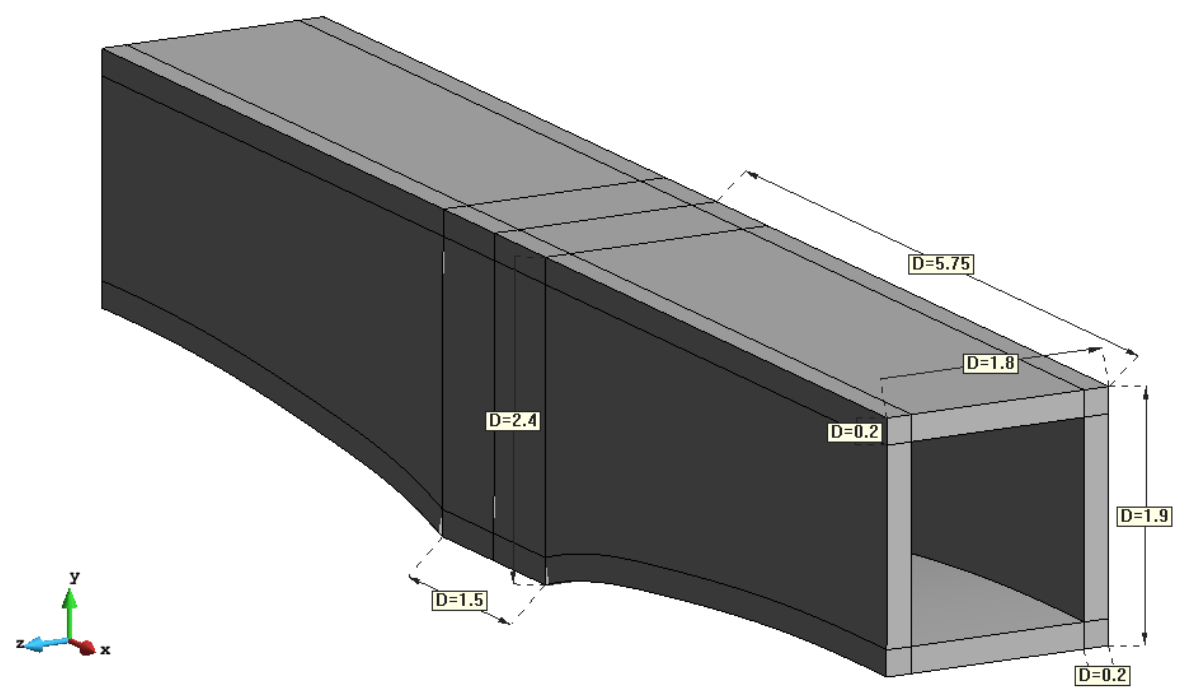

Fig. 27. Geometry of the cantilever

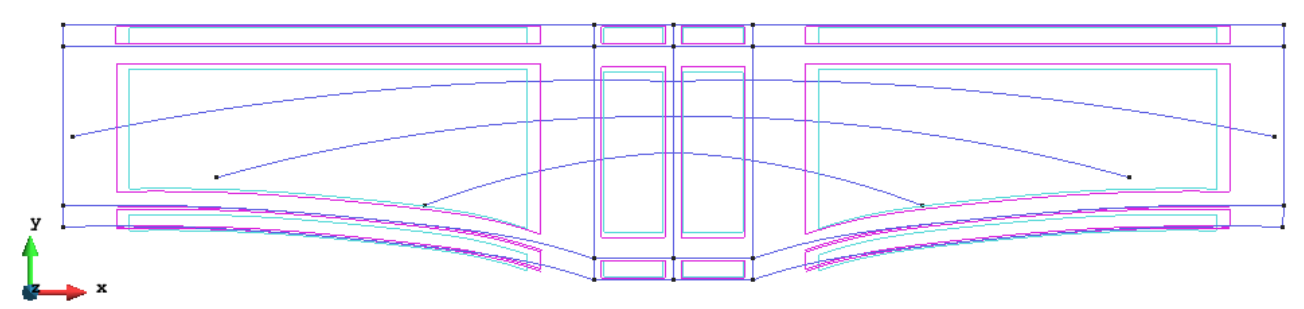

Fig. 28. Geometry of the cantilever and the steel tendons

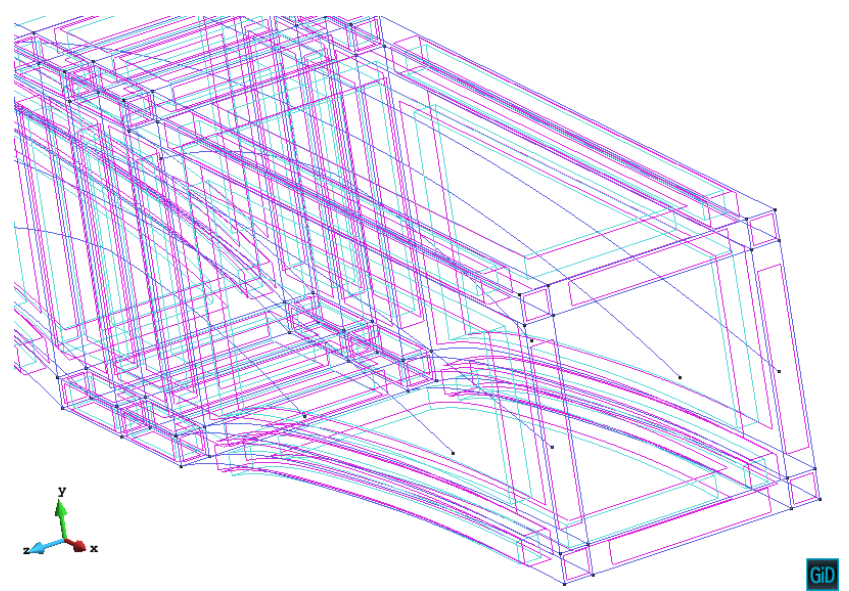

Fig. 29. Perspective of the cantilever and the steel tendons 
The finite element mesh shown in the Figs. 30-32 is composed by 196,928 linear hexahedra and 232,557 nodes. As depicted in the Figs. 30-32, the thickness of the structure has been discretized with 4 or 5 finite elements in order to capture properly the pre-stressing effects.

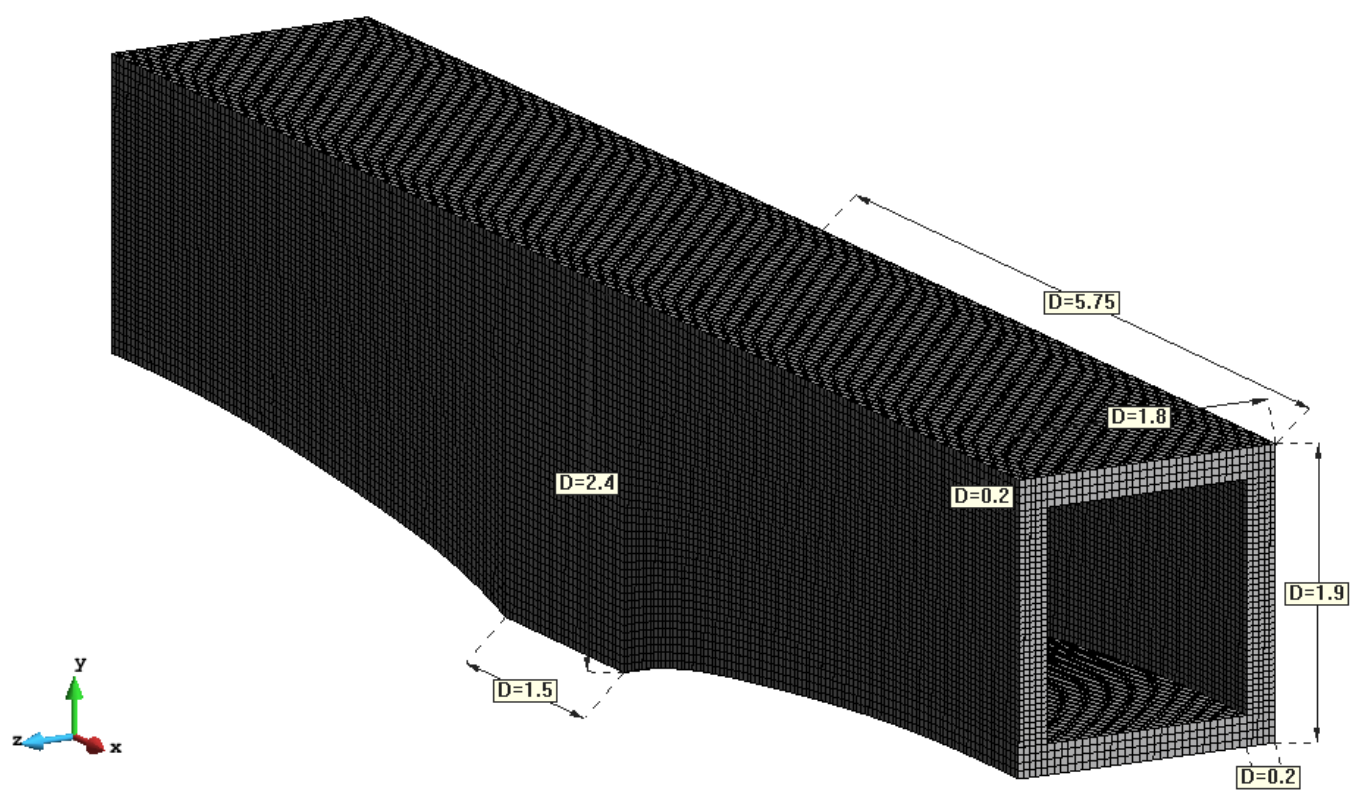

Fig. 30. Perspective of the finite element mesh (196,928 finite elements)

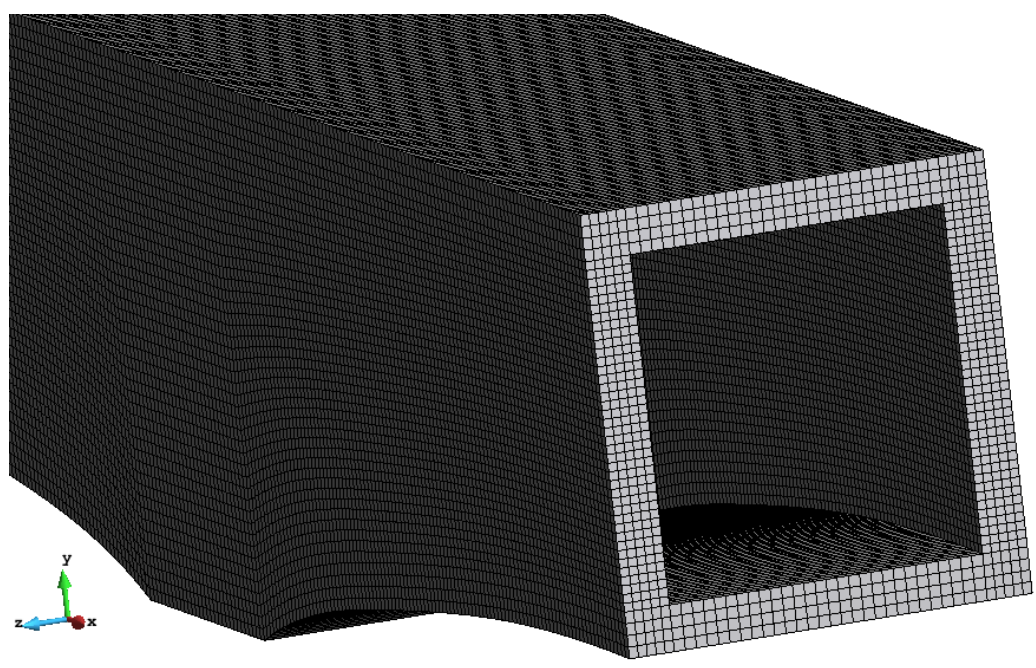

Fig. 31. Zoom of the inner part of the finite element mesh (196,928 finite elements)

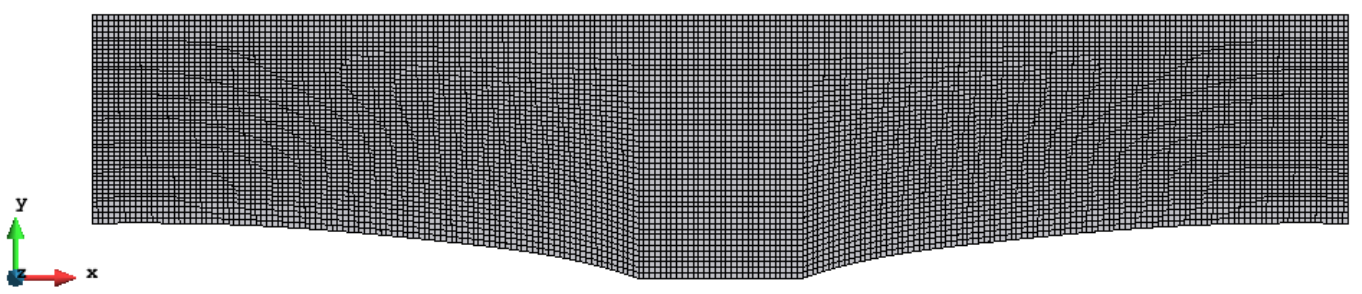

Fig. 32. x-y perspective of the finite element mesh (196,928 finite elements) 
After intersecting the linear elements that represent the steel tendons with the finite element mesh, we obtain a finite element discretization of the steel tendons inside the global mesh. The final result implies that there are some finite elements (the ones intersected by the active steel) with a certain volumetric participation and direction of pre-stressing whereas the rest behaves as a homogeneous concrete. The intersected finite elements can be seen in the Figs. 33-34. Each color symbolizes a different volumetric participation and/or different pre-stressing direction. In this case, 551 composite materials where generated.
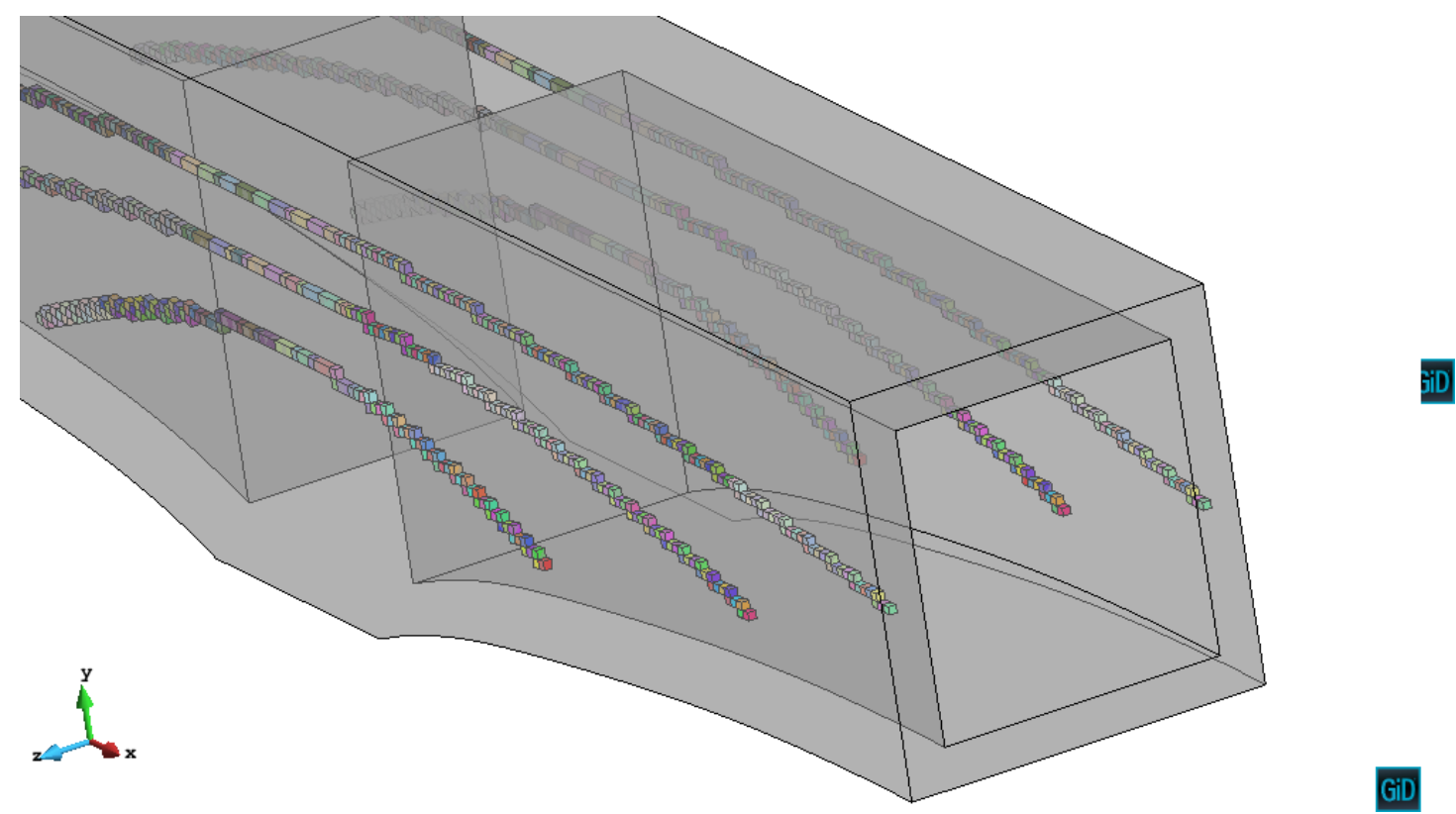

Fig. 33. Perspective of the finite elements intersected by the active steel

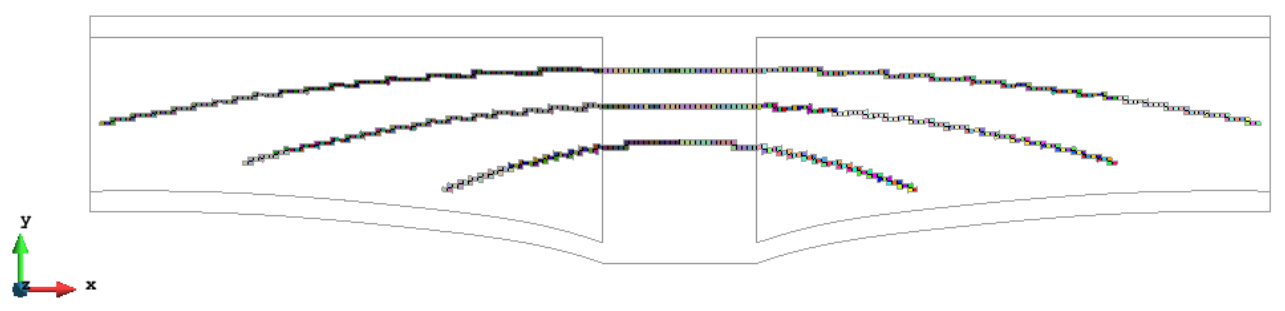

Fig. 34. x-y perspective of the finite elements intersected by the active steel

Next, the results of the computation of the structure neglecting the effect of the self-weight are shown. As in the previous case, the pre-stressing is performed in the initial year and then we let the active steel to relax up to 5 years.

In the Figs 35-36 one can see how the maximum displacement (at the tip of the cantilever) is reduced due to the viscoelasticity.

Analogously to the previous cases, the concrete is fully compressed and the active steel is in tension along its axis. Due to the viscoelasticity one can see how the principal tension stress of the active steel is reduced in time. 


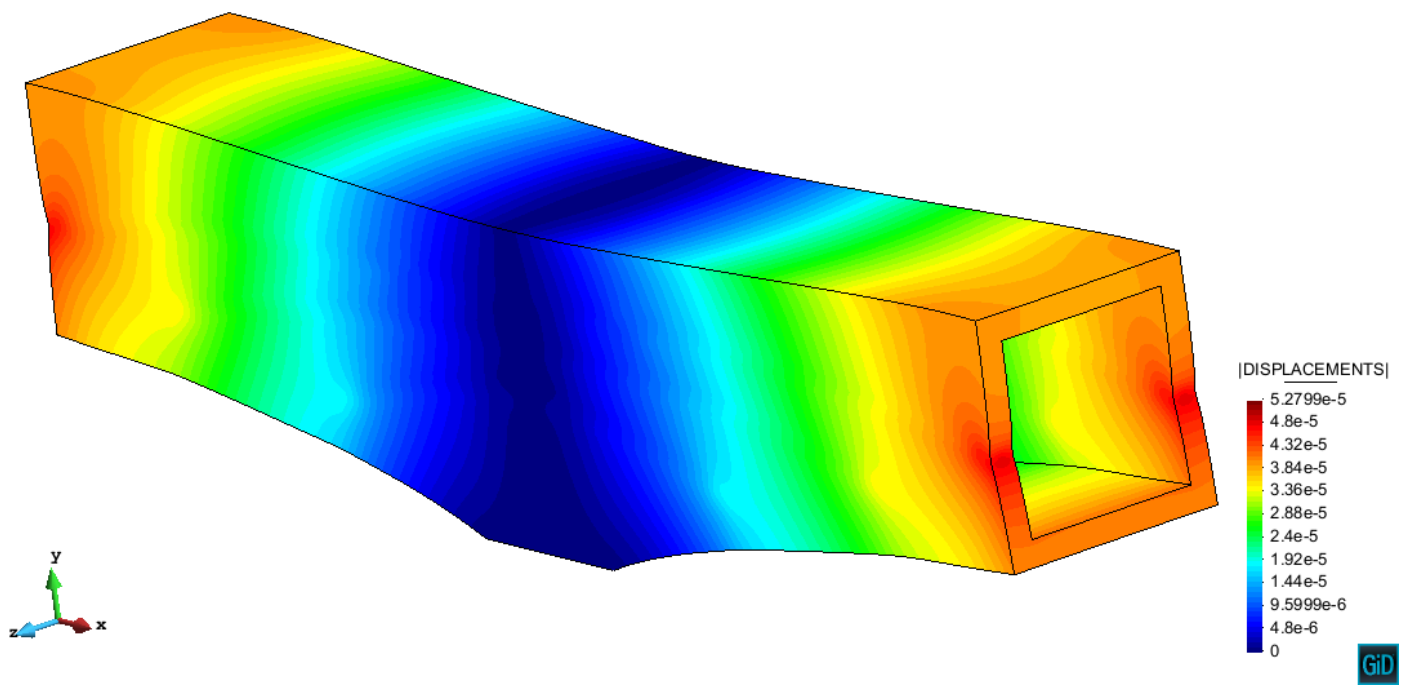

Fig. 35. Displacement path for the year $=0$, amplified 8000 times.

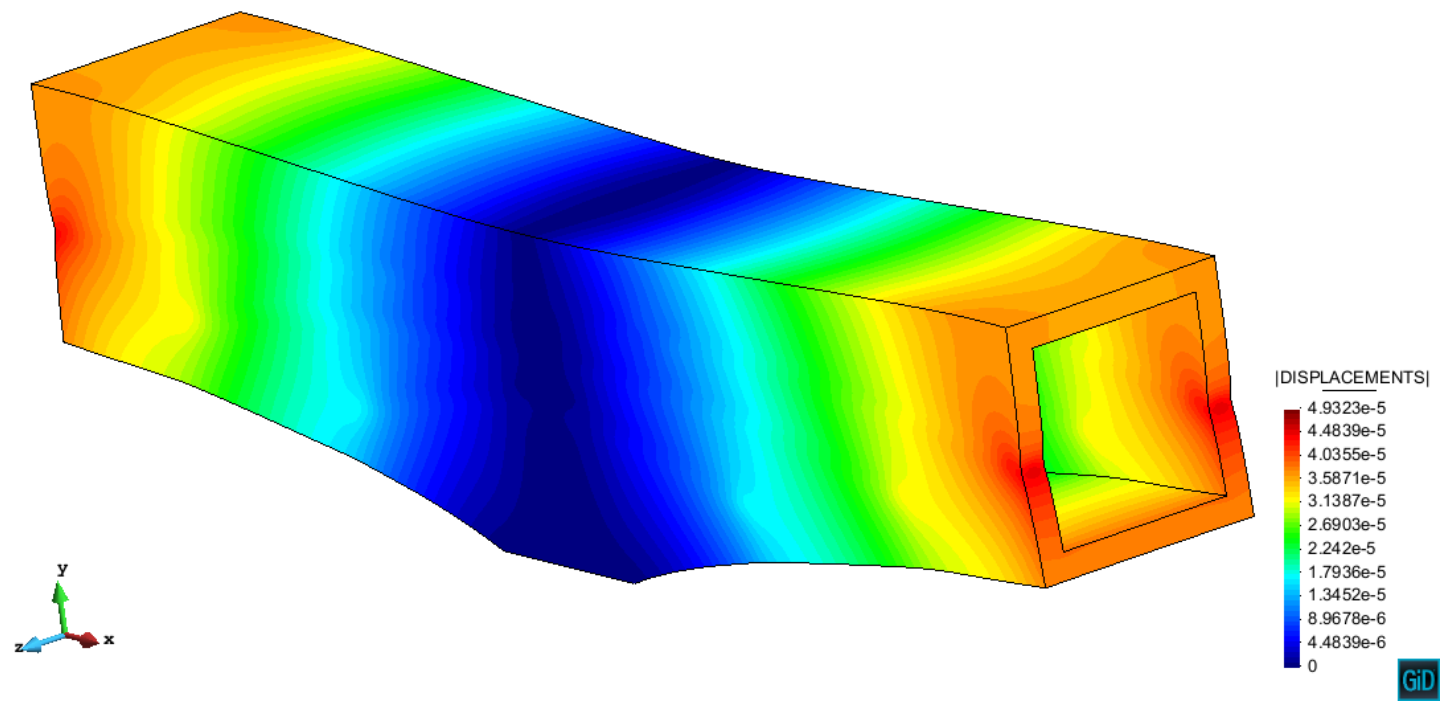

Fig. 36. Displacement path for the year $=5$, amplified 8000 times.

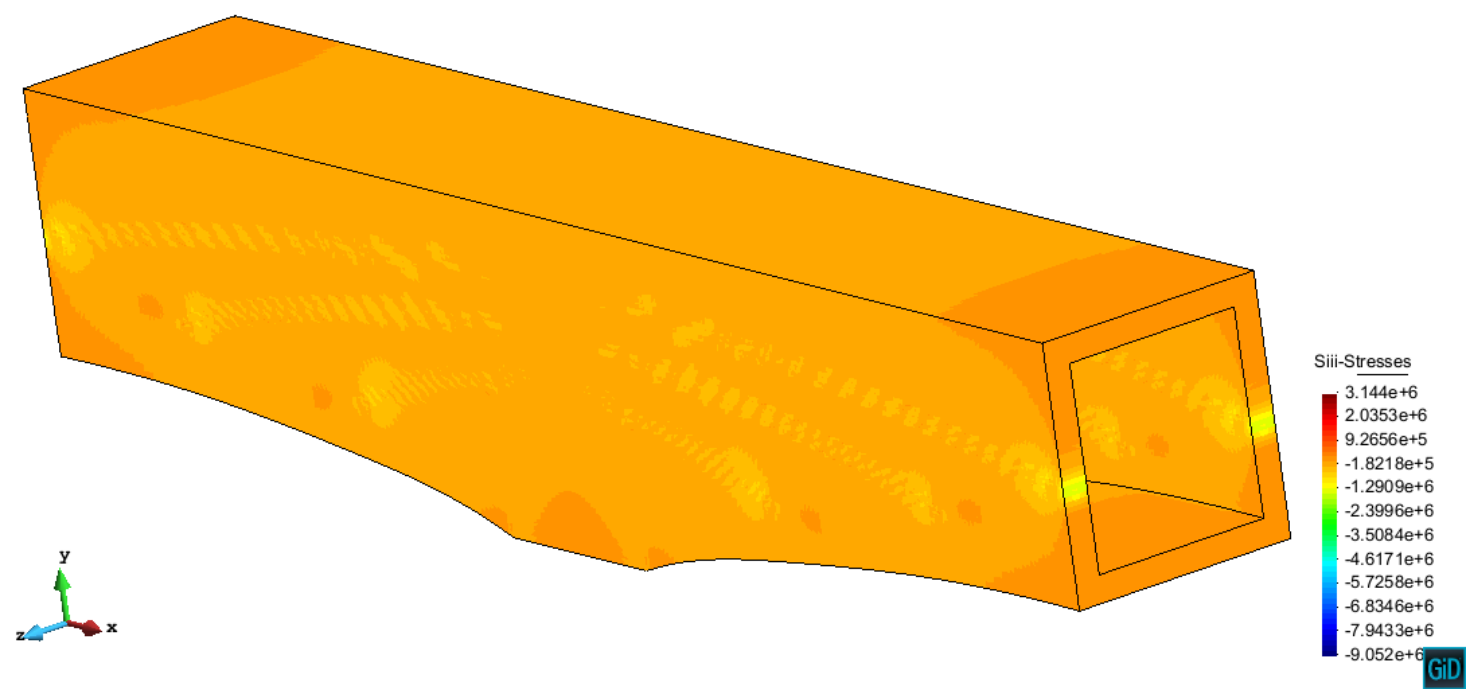

Fig. 37. Principal compression stress path for the concrete, year $=0$. 


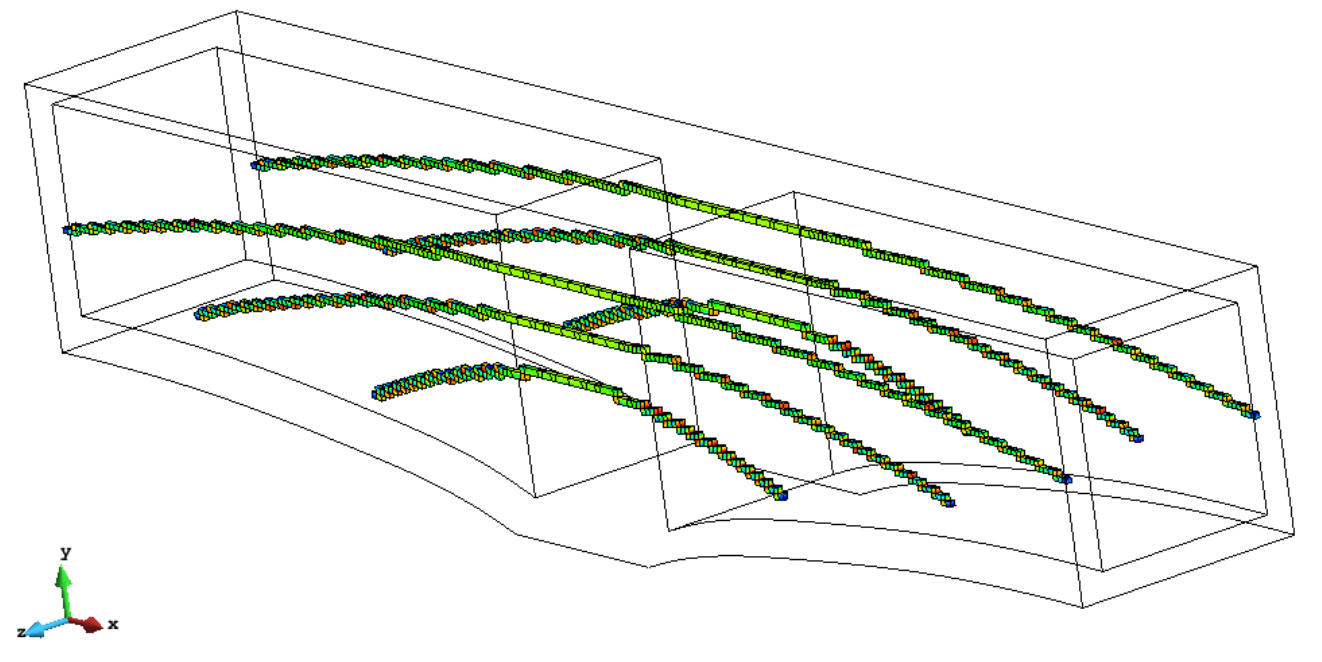

Fig. 38. Principal tension stress path for the active steel, year $=0$.

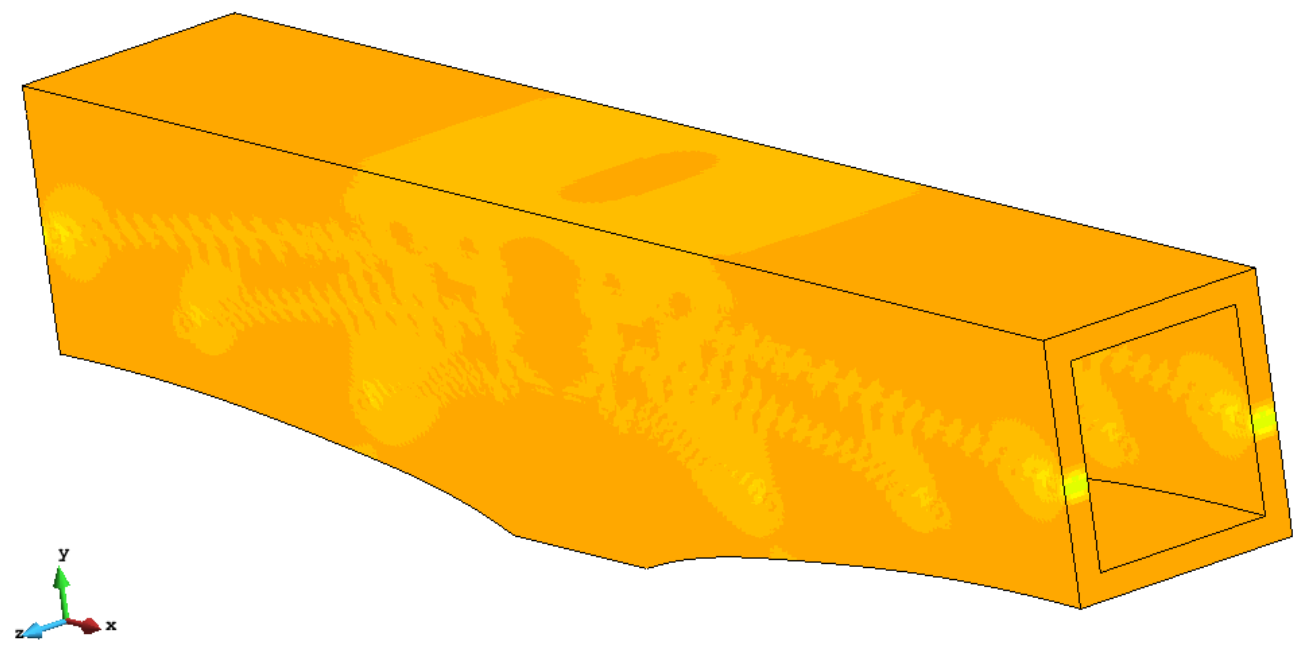

Fig. 39. Principal compression stress path for the concrete, year $=5$.

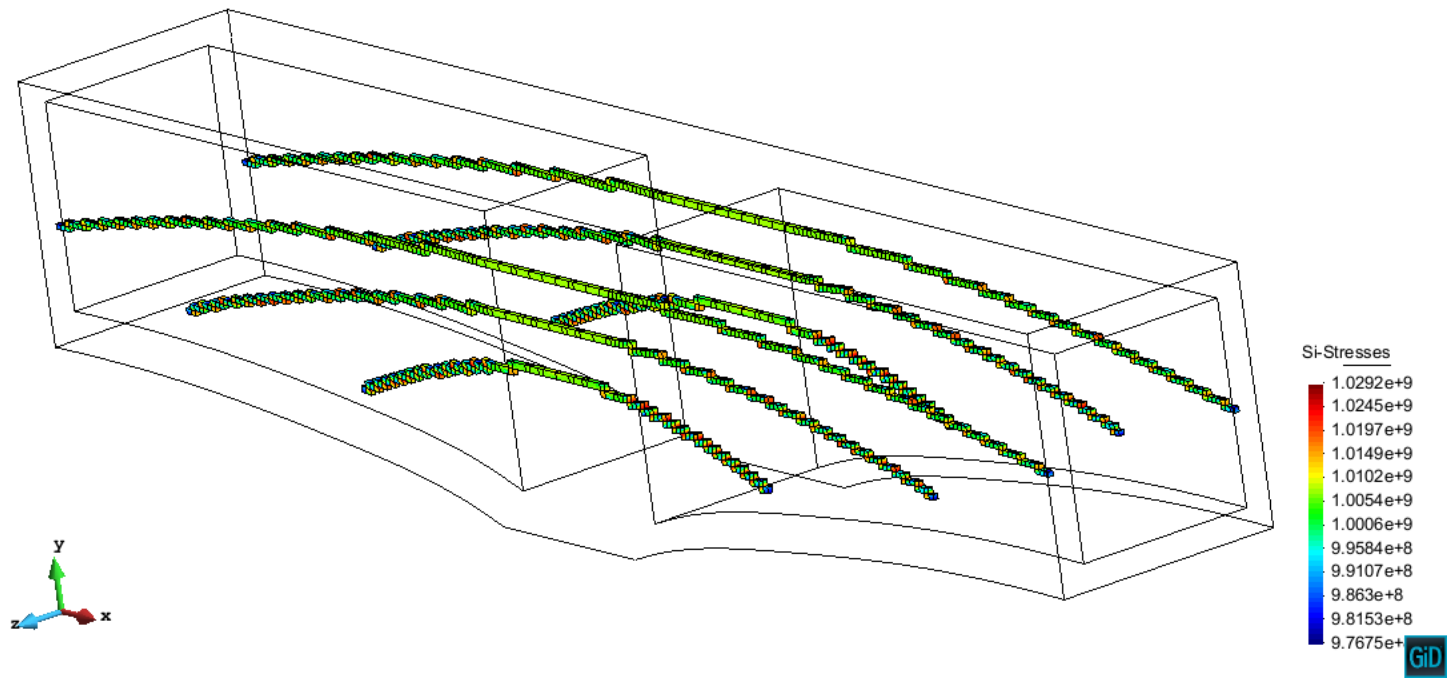

Fig. 40. Principal tension stress path for the active steel, year $=5$. 


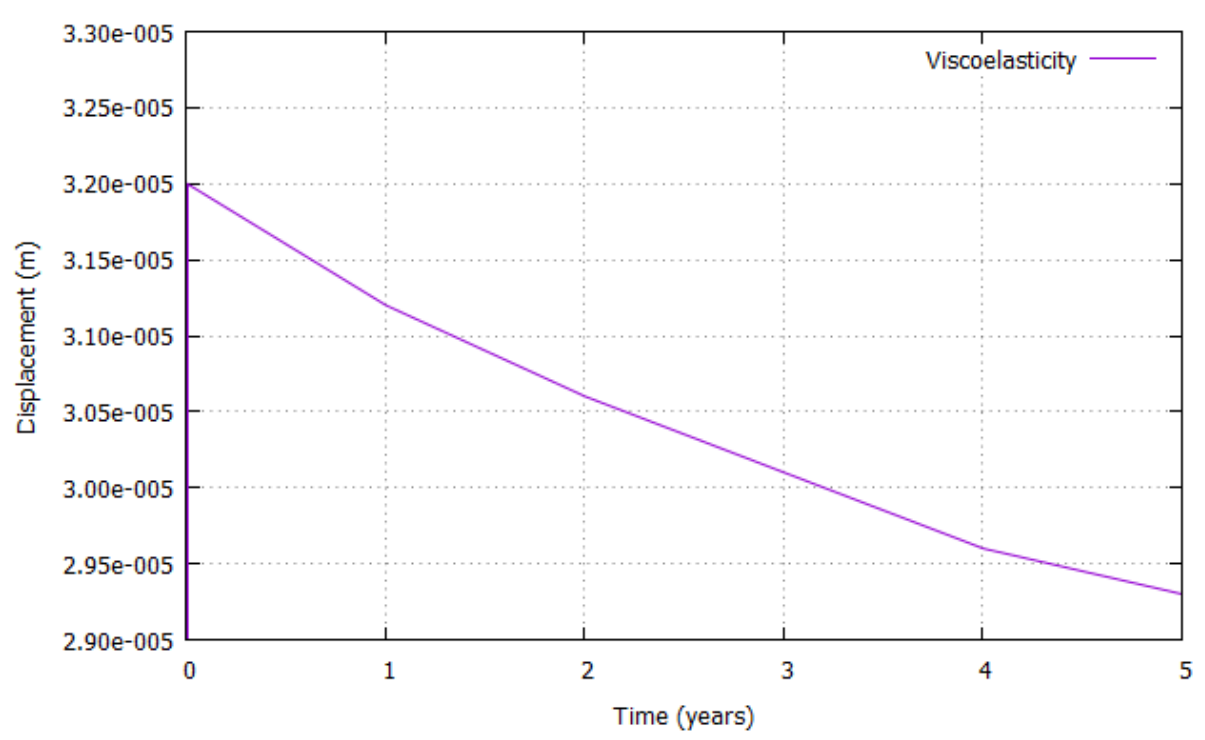

Fig. 41. Displacement evolution along time of the tip of the cantilever.

\subsection{Pre-stressed dome with 84 active steel tendons}

In this case, a $12 \mathrm{~m}$ height pre-stressed dome with 84 curvilinear steel tendons has been analysed. The geometry and its dimensions can be seen in the Figs. 42-43 whereas the linear description of the steel tendons is shown in the Figs. 44-45. The material properties and the pre-stressing data are analogous to the previous examples performed.

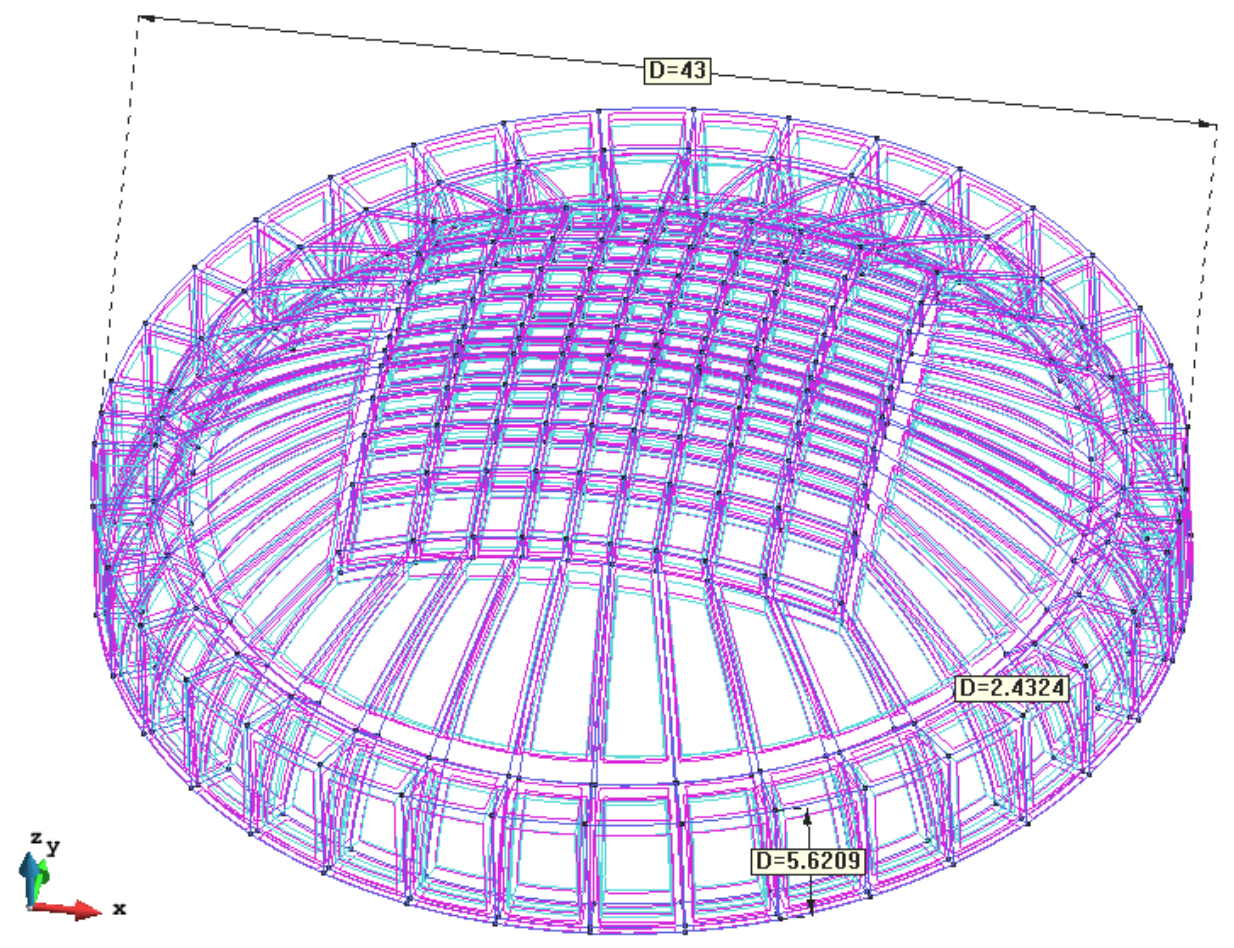

Fig. 42. Perspective of the geometry of the analyzed dome. 


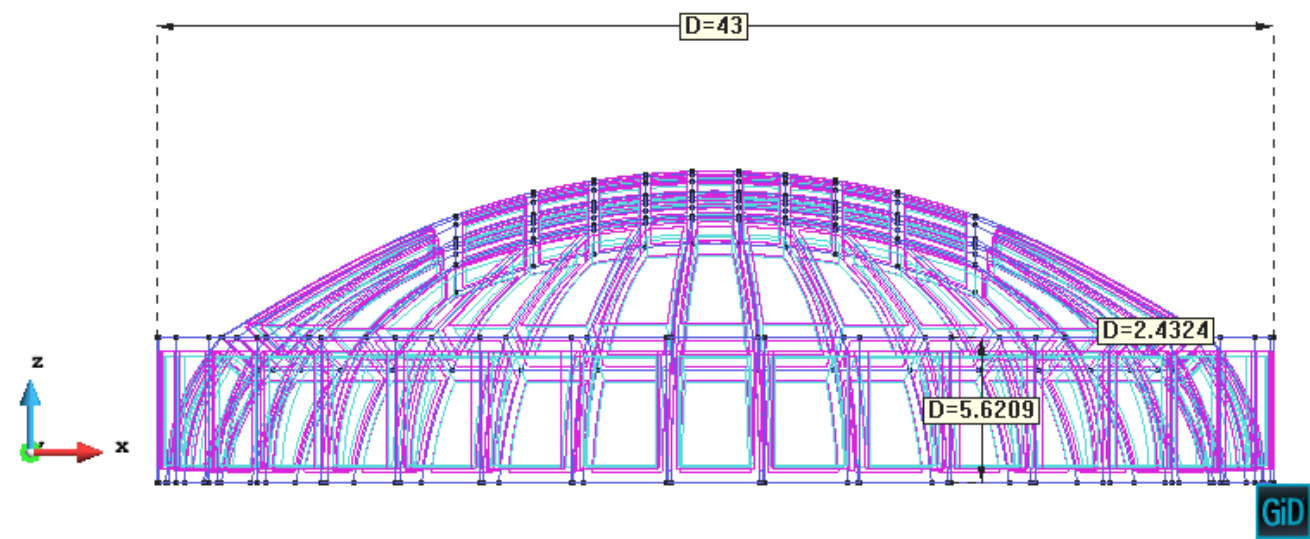

Fig.43. x-z view of the geometry of the analyzed dome.

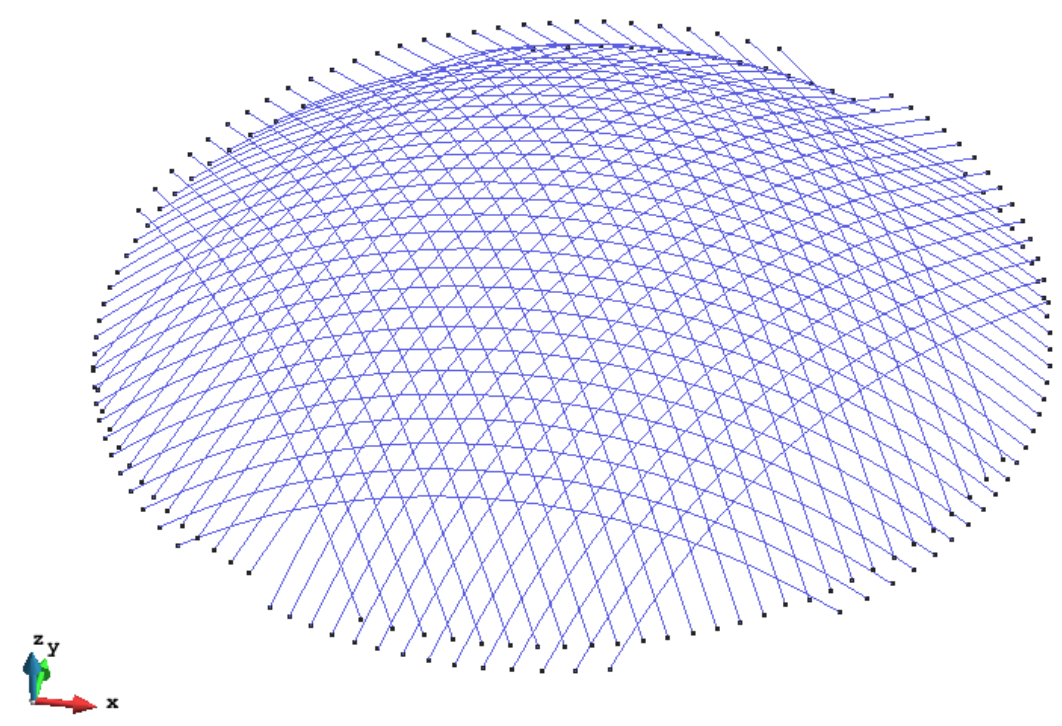

Fig. 44. Perspective of the geometry of the steel tendons.

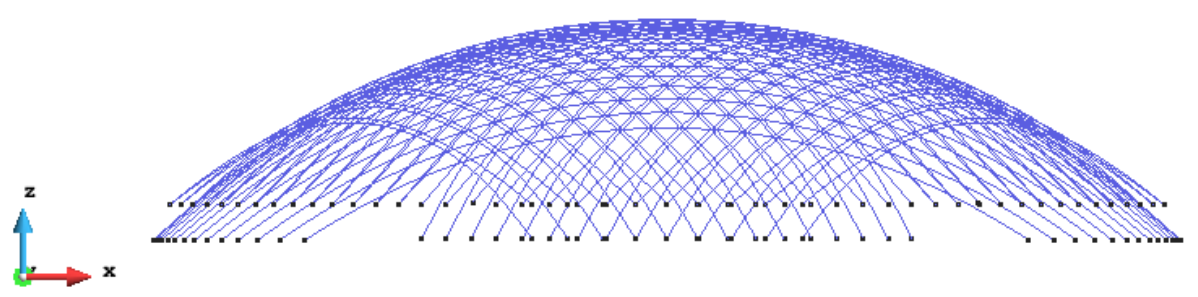

Fig. 45. $\mathrm{x}-\mathrm{z}$ view of the geometry of the steel tendons. 
The finite element mesh used can be seen in the Figs. 46-47 and is composed by 306,976 linear hexahedra and 351,078 nodes. After the intersection procedure of the lines with the finite element mesh, one can obtain an automatic generation of composite materials and, as depicted in the Figs. 48-50, its spatial localization. In the Figs. 48-50 one can see the position of the finite elements intersected by the steel tendons. These finite elements are going to be pre-stressed since they have a certain participation of active steel.

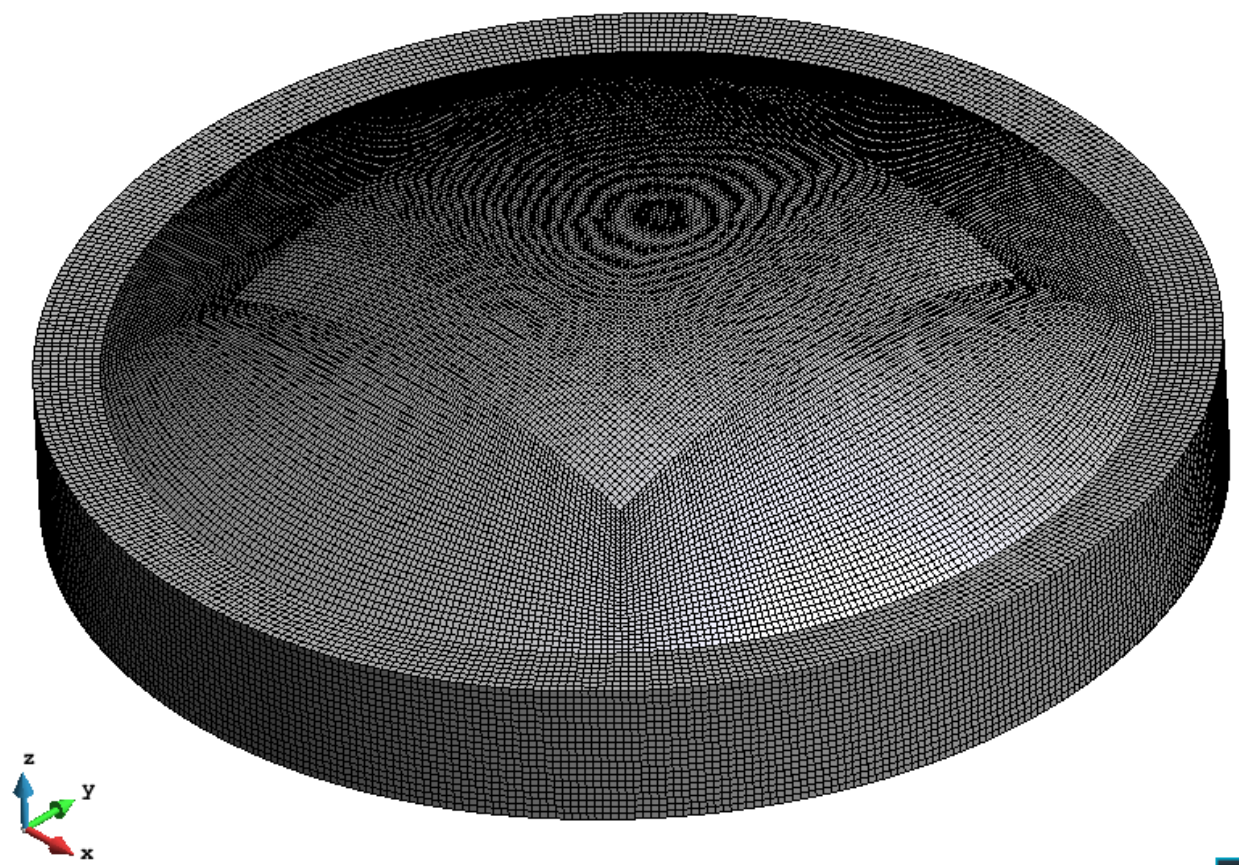

Fig. 46. Finite element mesh used (306,976 finite elements)

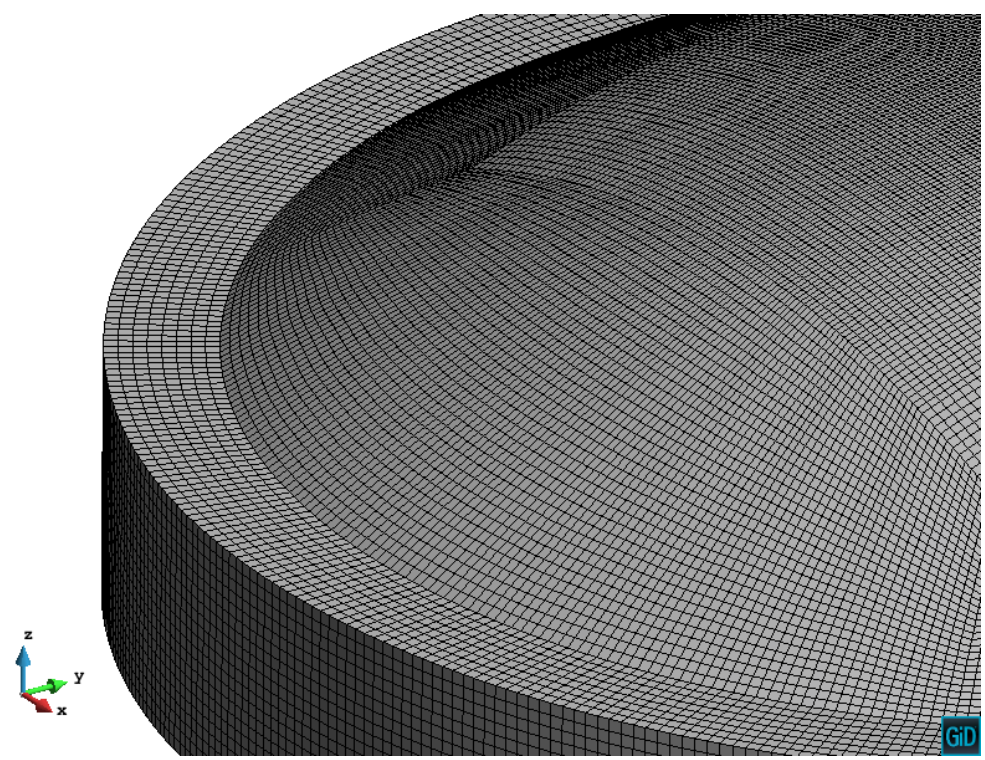

Fig. 47. Zoom of the finite element mesh used 


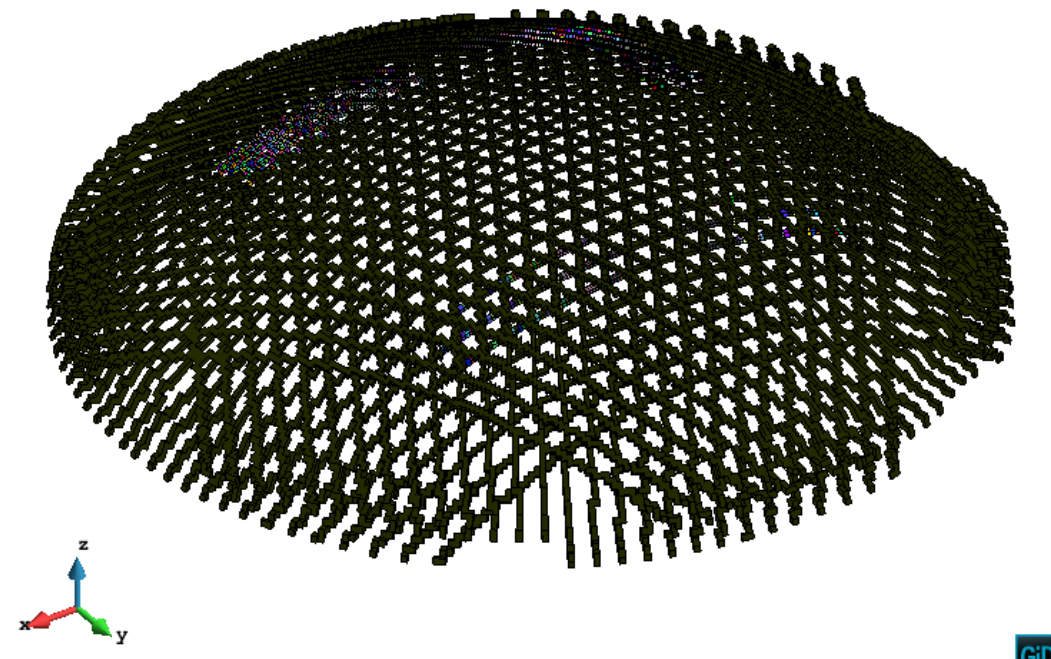

Fig. 48. Perspective of the finite element discretization of the steel tendons

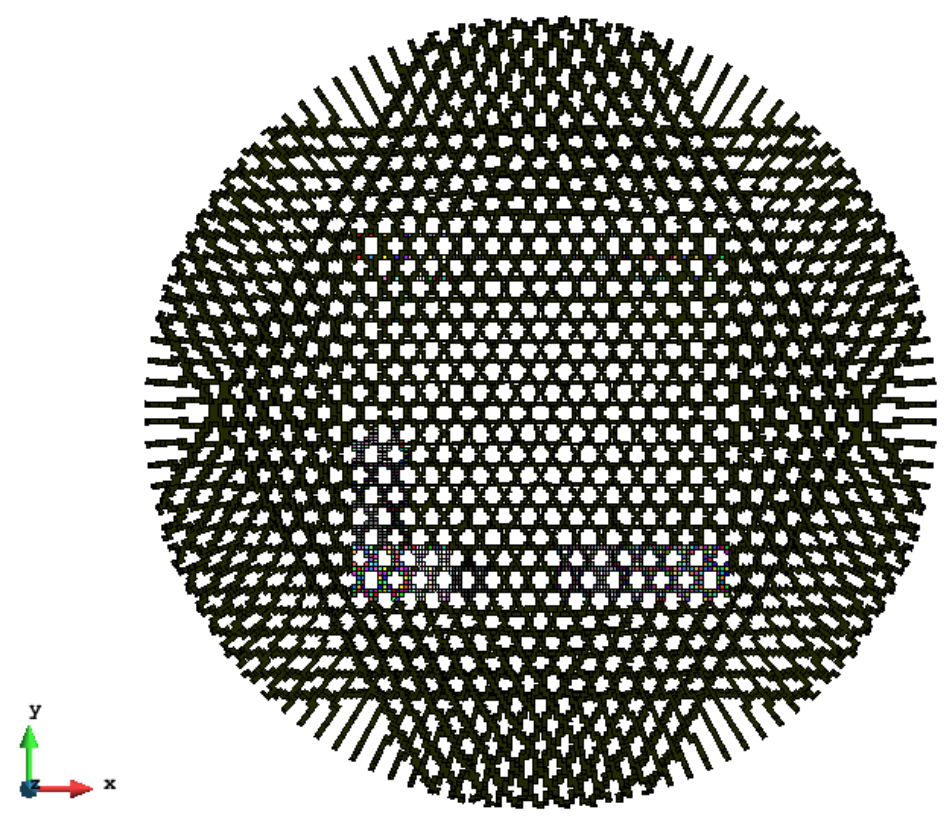

Fig. 49. $x-y$ view of the finite element discretization of the steel tendons

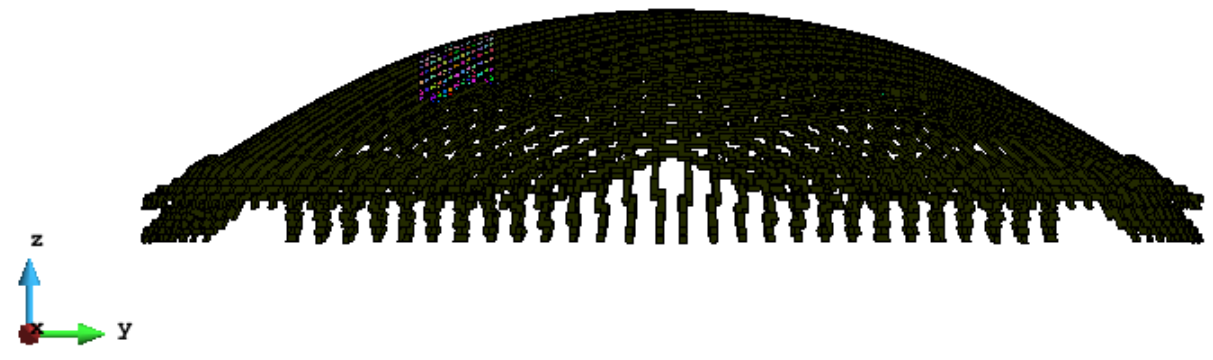

Fig. 50. z-y view of the finite element discretization of the steel tendons 
As in the previous examples, the pre-stressing is applied in the year 0 and, after that, the stress relaxation of the active steel takes place up to 5 years.

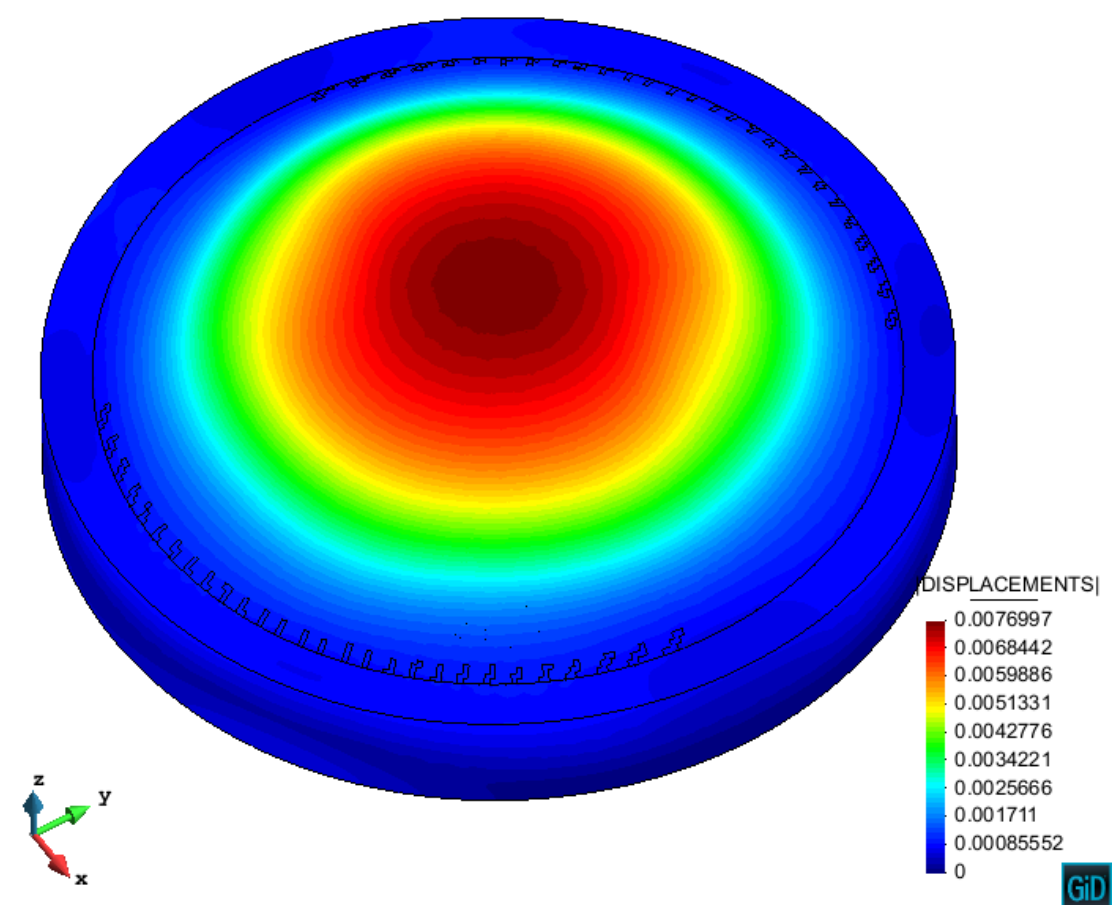

Fig. 51. Displacement path for the year 0.

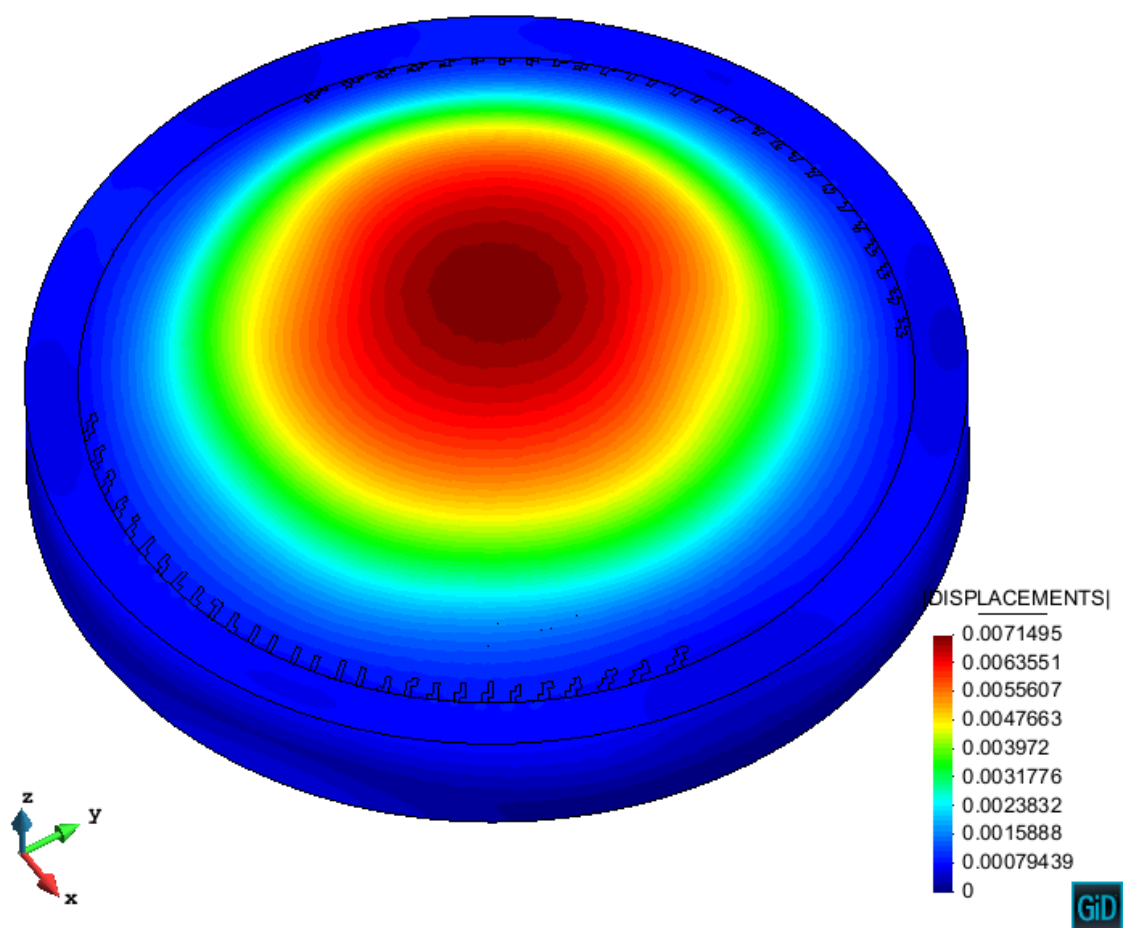

Fig. 52. Displacement path for the year 5 . 


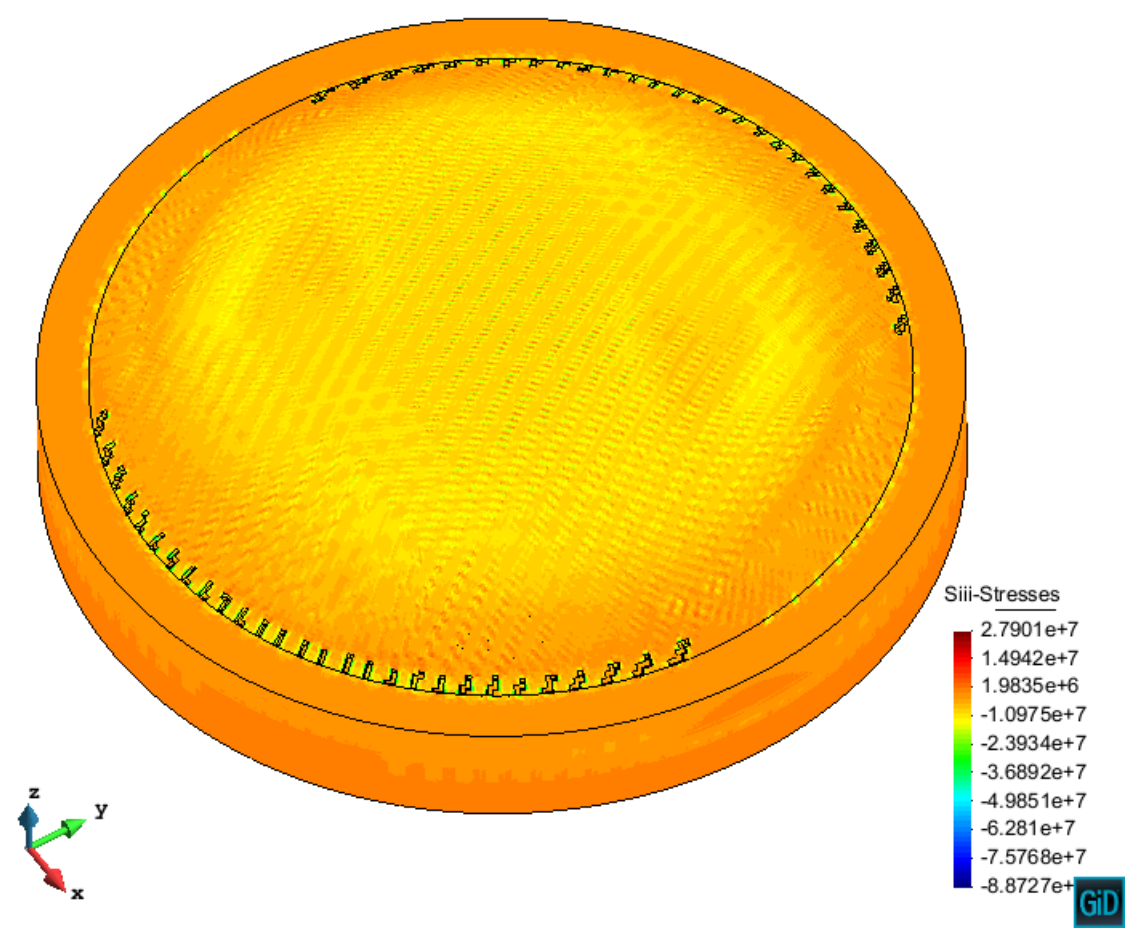

Fig. 53. Principal compression stress for the concrete, year 0.

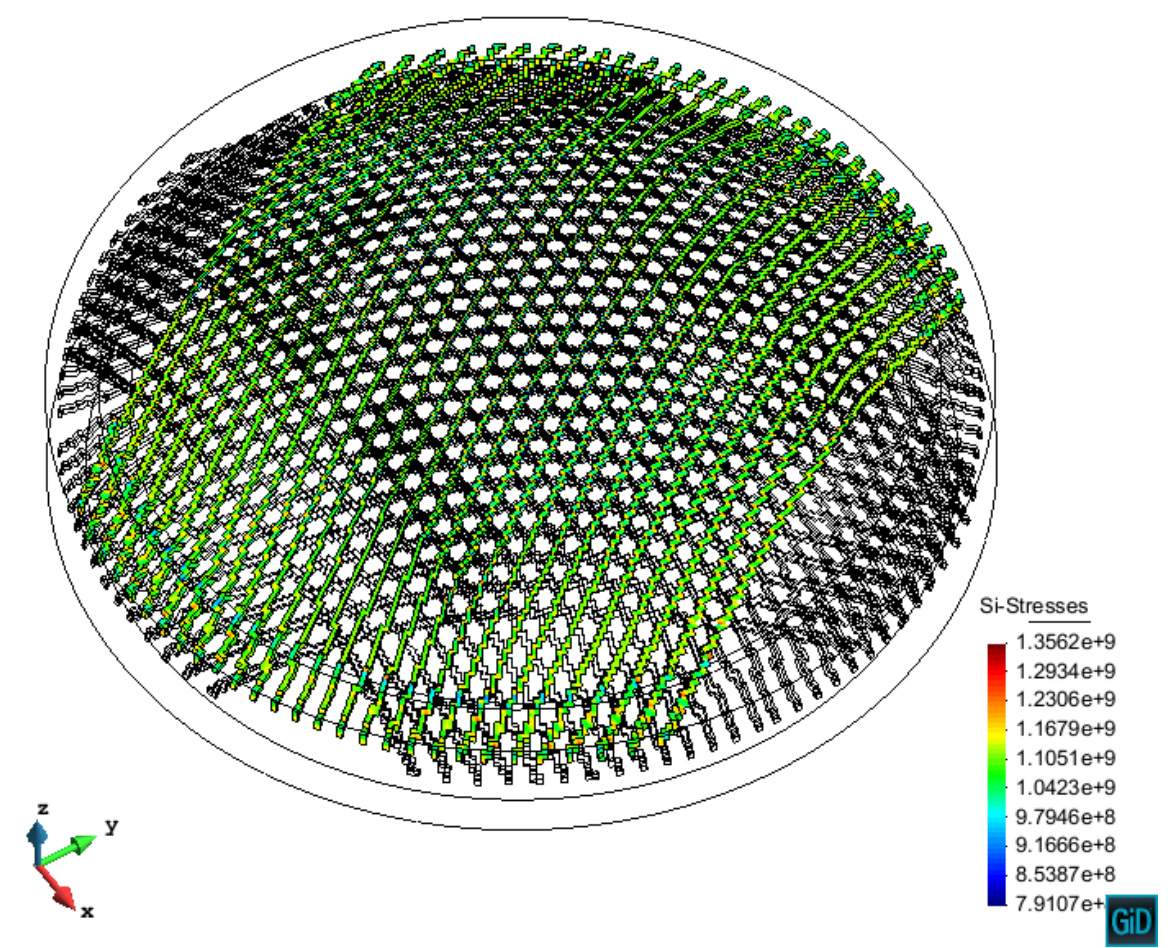

Fig. 54. Principal tension stresses for the active steel (one family), year 0. 


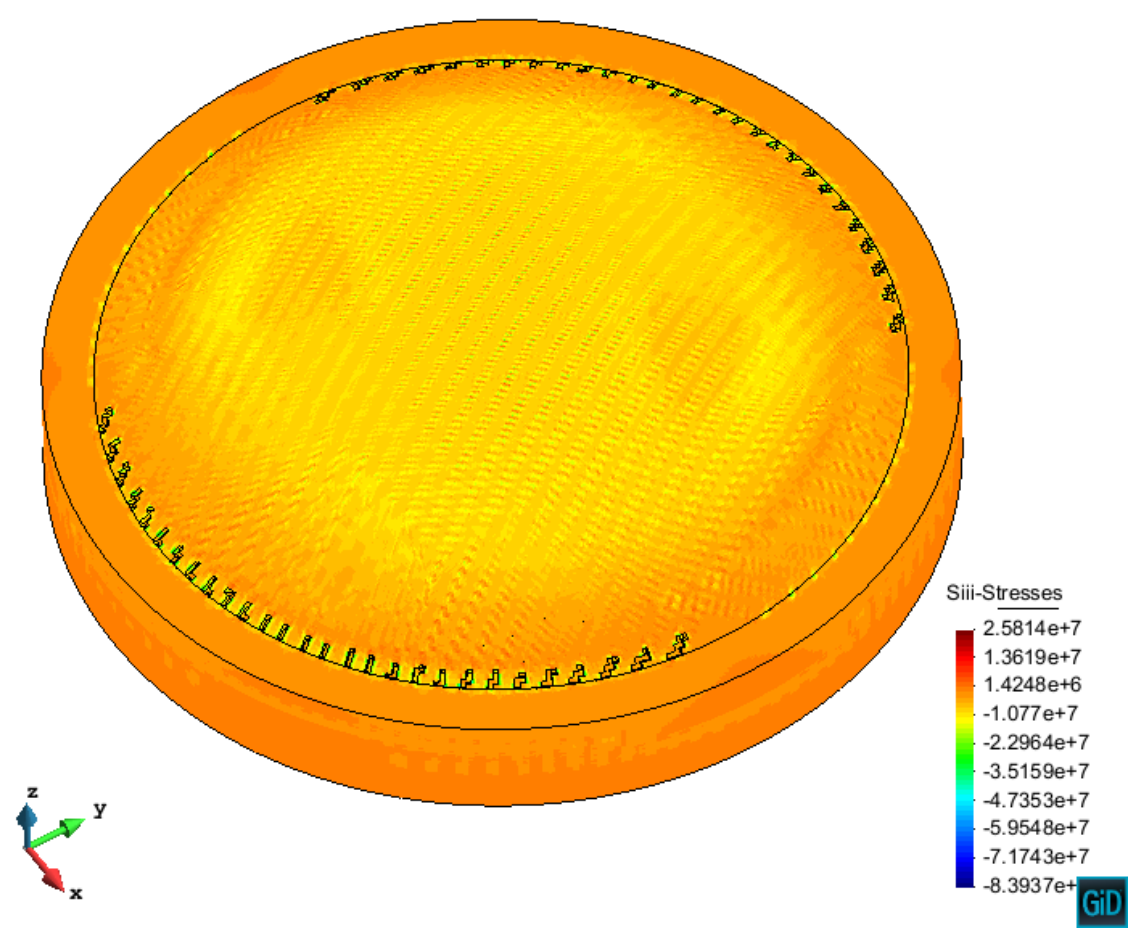

Fig. 55. Principal compression stress for the concrete, year 5.

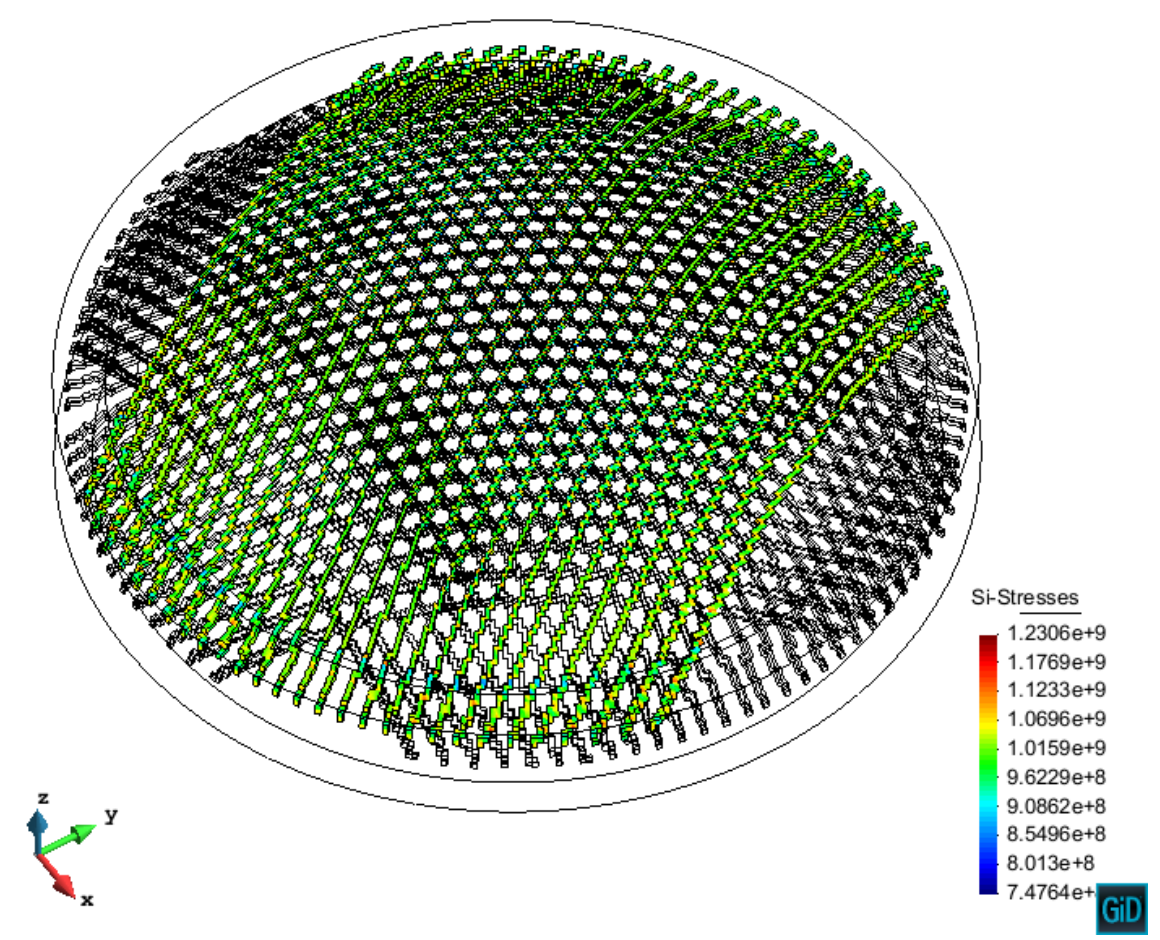

Fig. 56. Principal tension stresses for the active steel (one family), year 5. 


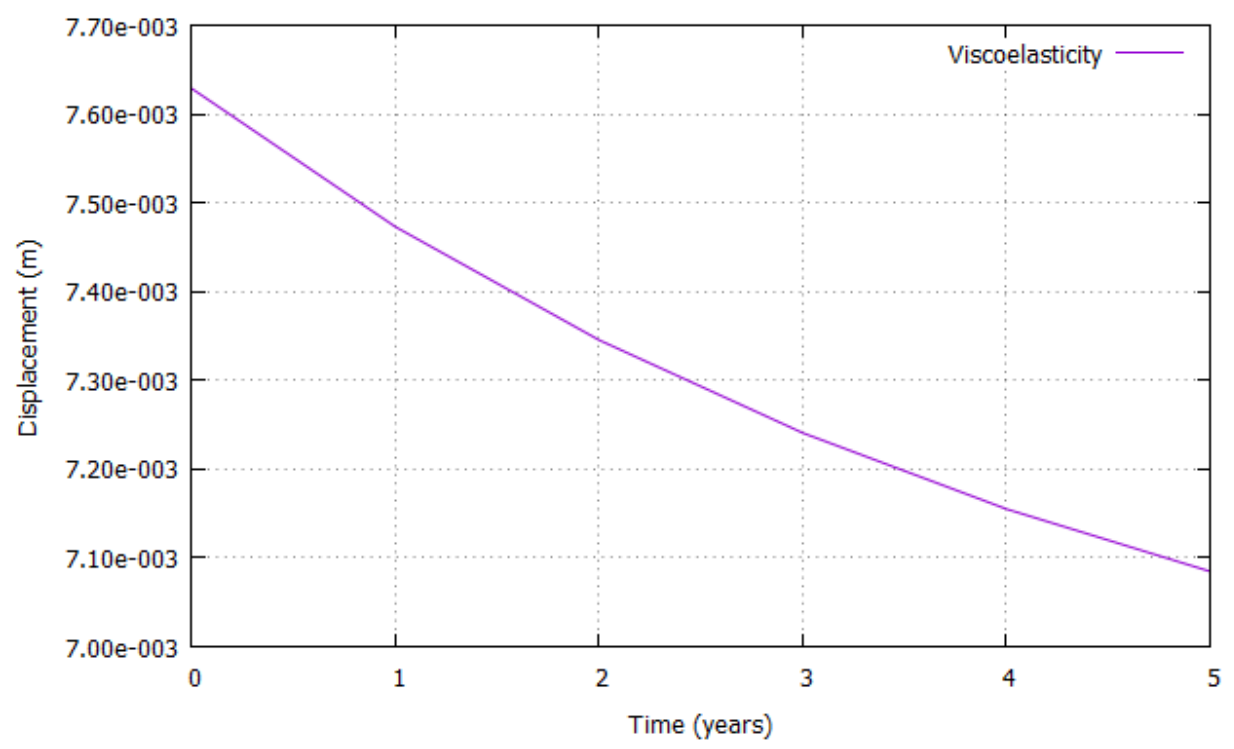

Fig. 57. Displacement evolution along time of the center of the dome.

\section{Conclusions}

This paper has presented a tool for performing the numerical analysis of pre-stressed and post-tensioned structures. The methodology has been validated by comparison with the analytical solution for very simple cases where the analytical solution is available.

Specifically, the longitudinal displacement due to the compression induced by the straight tendon in the first validation case has been computed by the FE analysis with a level of error of $10^{-5} \mathrm{~m}$ which implies a difference of $0.12 \%$. In the second validation case, the vertical displacement due to the parabolic tendon has been computed with an error of $5 \%$ assuming that the analytical solution is exact which, in fact, is false because the analytical solution does not include the effect of the shear stress and the elastic shortening of the concrete.

Three other cases have been shown, of three different structures with varying levels of complexity. They serve as an example of industrial application on real life structures that can be commonly found in the field of civil engineering. With this methodology results can be obtained on large scale structures that have up to 84 steel tendons, as shown in one of the examples. However, the number of tendons is not a limitation of this modelling procedure.

We can therefore conclude that the formulation is capable of simulating the effect of the pre-stressing of tendons in any type of structure with any shape and/or boundary condition.

Acknowledgements: This work has been partially supported by the Spanish "Ministerio de Economía y Competividad" through the projects BIA2015-67807-R - RESCICLO and EUIN2015-62818 - RESSAFE and by the European Commission by means of the project TCAiNMaND- PIRSES-GA-2013612607.

\section{References}

[1] Rastellini, F., Oller, S., Salomon, O., Oñate, E., 2008. Composite materials non-linear modelling for long fibre reinforced laminates: Continuum basis, computational aspects and validations. Computers and Structures 86(9), 879-896 
[2] Martinez, X., Oller, S., Rastellini, F. and Barbat, A.H., 2008. A numerical procedure simulating RC structures reinforced with FRP using the serial/parallel mixing theory, Computers and Structures, 86(1516):1604-18.

[3] Martinez, X., Oller, S., Rastellini, F. Análisis no-lineal de materiales compuestos mediante la teoría de mezclas serie-paralelo. Omnia Publisher, 2014. Capítulo 10 - p. 237-260. Libro: Aplicaciones avanzadas de los materiales compuestos en la obra civil y la edificación, (http://dx.doi.org/10.3926/oms.209)

[4] Rezaie, F., Famam, S.M. Fracture mechanics analysis of pre-stressed concrete sleepers via investigating crack initiation length. Engineering Failure Analysis 58 (2015) 267-280.

[5] Shokoohfar, A., Rahai, A. Nonlinear analysis of pre-stressed concrete containment vessel (PCCV) using the damage plasticity model. Nuclear Engineering and Design 298 (2016) 41-50.

[6] Tavakkoli I, Kianoush MR, Abrishami H, Han X, Finite element modelling of a nuclear containment structure subjected to high internal pressure, International Journal of Pressure Vessels and Piping (2017), doi: 10.1016/j.ijpvp.2017.05.004

[7] A.K.H. Kwan, F.J. Ma, Crack width analysis of reinforced concrete under direct tension by finite element method and crack queuing algorithm, In Engineering Structures, Volume 126, 2016, Pages 618-627,

[8] Amir A. Arab, Sameh S. Badie, Majid T. Manzari, A methodological approach for finite element modeling of pretensioned concrete members at the release of pretensioning, In Engineering Structures, Volume 33, Issue 6, 2011, Pages 1918-1929

[9] Kaewunruen S, Remennikov A. Nonlinear finite element modeling of railway prestressed concrete sleeper. In: 10th East Asia-Pacific conference on structural engineering and construction. Proceedings. Real structures: bridges and tall buildings. vol. 4. 2006. p. 323-8.

[10] Stephen E. Simulation of the long-term behavior of precast/prestressed concrete bridges. Master of science thesis. University of Cincinnati. Department of Civil and Environmental Engineering. 2006.

[11] T. Rabczuk, J. Eibl Numerical analysis of prestressed concrete beams using a coupled element free Galerkin/finite element approach Internat J Solids Structures, 41 (2004), pp. 1061-1080

[12] A. Ayoub, F. Filippou Finite-element model for pretensioned prestressed concrete girders J Struct Eng, 41 (2010), pp. 401-409

[13] Hu, H.T., and Lin, Y.H., Ultimate analysis of PWR prestressed concrete containment subjected to internal pressure, International journal of pressure vessels and piping, 83.3 (2006):161-167.

[14] Spanish Minister of Public Works. Instrucción de Hormigón Estructural EHE-08 (Spanish Structural Concrete Code); 2008.

[15] Oller, S. Dinamica no-lineal, CIMNE, Nro. 63, Barcelona, Spain, 2003. ISBN 84-89925-95-X

[16] PLCd Manual. Non-linear thermo-mechanic finite element code oriented to PhD student education. Code developed at CIMNE; 1991-to present. URL: 〈http://www.cimne.com/plcd>. 